\title{
Pacific
}

Journal of

Mathematics

\section{$K(n)$-LOCALIZATION OF THE $K(n+1)$-LOCAL $E_{n+1}$-ADAMS SPECTRAL SEQUENCES}

TAKESHI TORII

Volume $250 \quad$ No. 2

April 2011 


\title{
$K(n)$-LOCALIZATION OF THE $K(n+1)$-LOCAL $E_{n+1}$-ADAMS SPECTRAL SEQUENCES
}

\author{
TAKESHI TORII
}

\begin{abstract}
We construct a spectral sequence converging to the homotopy set of maps from a spectrum to the $K(n)$-localization of the $K(n+1)$-local sphere. We also construct a map of spectral sequences from the $K(n)$-local $E_{n}$-Adams spectral sequence to the preceding one. Then we compare the map on $E_{2}$-terms with a map induced by the inflation maps of continuous cohomology groups for Morava stabilizer groups. As an application we show that $\zeta_{n}$ in $\pi_{-1}\left(L_{K(n)} S^{0}\right)$ represented by the reduced norm map in the $K(n)$ local $E_{n}$-Adams spectral sequence has a nontrivial image under the map $\pi_{*}\left(L_{K(n)} S^{0}\right) \rightarrow \pi_{*}\left(L_{K(n)} L_{K(n+1)} S^{0}\right)$.
\end{abstract}

\section{Introduction}

The motivation of this note is toward understanding the relationship between the $K(n)$-local category and the $K(n+1)$-local category. For each prime number $p$, the stable homotopy category of $p$-local spectra has a filtration of full subcategories corresponding to the height filtration of the moduli space of formal groups [Morava 1985]. The $n$-th associated graded part of the filtration is equivalent to the $K(n)$-local category, that is, the Bousfield localization of the stable homotopy category with respect to the $n$-th Morava $K$-theory spectrum $K(n)$ [Hovey and Strickland 1999]. So it can be considered that the stable homotopy category of $p$-local spectra is built up from the $K(n)$-local categories for various $n$. In fact, the chromatic convergence theorem [Ravenel 1992] says that a $p$-local finite spectrum $X$ is homotopy equivalent to the homotopy inverse limit of the chromatic tower $\cdots \rightarrow L_{n+1} X \rightarrow L_{n} X \rightarrow \cdots \rightarrow L_{0} X$, where $L_{n}$ is the Bousfield localization functor with respect to the wedge of Morava $K$-theories $K(0) \vee K(1) \vee \cdots \vee K(n)$. This means that a $p$-local finite spectrum $X$ can be recovered from $\left\{L_{n} X\right\}_{n \geq 0}$ through the chromatic tower. Furthermore, if the chromatic splitting conjecture is true, then it implies that the $p$-completion of a finite spectrum $X$ is a direct summand of the product $\prod_{n} L_{K(n)} X$ [Hovey 1995]. This means that it is not necessary to reconstruct the tower but it is sufficient to know all $L_{K(n)} X$ to obtain

MSC2000: primary 55T25, 55P42; secondary 55Q51, 55N22, 55N20.

Keywords: Adams spectral sequence, $K(n)$-localization, Morava $E$-theory. 
some information of $X$. Since the chromatic splitting conjecture is concerned with the relationship among various chromatic pieces, it is important to understand the relationship between the $K(n)$-local category and the $K(n+1)$-local category.

Let $E_{n}$ be the $n$-th Morava $E$-theory spectrum. The $K(n)$-local $E_{n}$-Adams spectral sequence $L_{K(n)} E_{r}^{s, t}(W)$ is a natural spectral sequence for any spectrum $W$,

$$
L_{K(n)} E_{2}^{s, t}(W)=H_{c}^{s}\left(G_{n} ; E_{n}^{t}(W)\right) \Longrightarrow\left[W, L_{K(n)} S^{0}\right]^{s+t},
$$

which converges to [W, $\left.L_{K(n)} S^{0}\right]^{*}$ strongly and conditionally; see [Devinatz and Hopkins 2004, Appendix A]. On the $E_{2}$-term, $G_{n}$ is the $n$-th extended Morava stabilizer group, and $H_{c}^{s}\left(G_{n} ; E_{n}^{t}(W)\right)$ is a continuous cohomology group for the profinite group $G_{n}$ with coefficients in the profinite module $E_{n}^{t}(W)$.

We construct a natural spectral sequence converging to $\left[W, L_{K(n)} L_{K(n+1)} S^{0}\right]^{*}$ by applying the $K(n)$-localization functor to the $K(n+1)$-local $E_{n+1}$-Adams resolution of $L_{K(n+1)} S^{0}$. Let $\mathbb{A}=L_{K(n)} E_{n+1}$ be the $K(n)$-localization of the $(n+1)$-st Morava $E$-theory $E_{n+1}$. We identify the $E_{2}$-term as a cohomology group based on the continuous cochain complex for $G_{n+1}$ with coefficients in the topological module $\mathbb{A}^{*}(W)$. We call this spectral sequence the $K(n)$-localization of the $K(n+1)$-local $E_{n+1}$-Adams spectral sequence for $W$.

Theorem 4.7. For any spectrum $W$, there is a natural spectral sequence

$$
L_{K(n)} L_{K(n+1)} E_{2}^{s, t}(W)=H_{c}^{s}\left(G_{n+1} ; \mathbb{A}^{t}(W)\right) \Longrightarrow\left[W, L_{K(n)} L_{K(n+1)} S^{0}\right]^{s+t},
$$

which converges strongly and conditionally.

By the $K(n)$-localization of the $K(n+1)$-localization map $S^{0} \rightarrow L_{K(n+1)} S^{0}$, we obtain a map $L_{K(n)} S^{0} \rightarrow L_{K(n)} L_{K(n+1)} S^{0}$, which induces a map

$$
\left[W, L_{K(n)} S^{0}\right]^{*} \rightarrow\left[W, L_{K(n)} L_{K(n+1)} S^{0}\right]^{*}
$$

for any spectrum $W$. We construct in Theorem 6.2 a natural map of spectral sequences

$$
\varphi_{r}(W): L_{K(n)} E_{r}^{s, t}(W) \longrightarrow L_{K(n)} L_{K(n+1)} E_{r}^{s, t}(W),
$$

which converges to the map $\left[W, L_{K(n)} S^{0}\right]^{s+t} \rightarrow\left[W, L_{K(n)} L_{K(n+1)} S^{0}\right]^{s+t}$. Furthermore, we give an interpretation of the map on $E_{2}$-terms. We construct a natural homomorphism

$$
\theta(W): H_{c}^{*}\left(G_{n} ; E_{n}^{*}(W)\right) \longrightarrow H_{c}^{*}\left(G_{n+1} ; \mathbb{A}^{*}(W)\right),
$$

which is obtained from some kind of inflation maps (see (7-1)).

Theorem 7.6. The map $\varphi_{2}(W)$ coincides with $\theta(W)$ for any spectrum $W$.

By the Hopkins-Miller theorem [Devinatz and Hopkins 2004, Theorem 6], we know that there is a nontrivial element $\zeta_{n} \in \pi_{-1}\left(L_{K(n)} S^{0}\right)$ which is represented by 
the reduced norm map of $G_{n}$ in the $E_{2}$-term of the $K(n)$-local $E_{n}$-Adams spectral sequence. Let $\omega_{n}$ be the image of $\zeta_{n}$ under the map

$$
\pi_{*}\left(L_{K(n)} S^{0}\right) \rightarrow \pi_{*}\left(L_{K(n)} L_{K(n+1)} S^{0}\right) .
$$

As an application of our results, we show the following theorem.

\section{Theorem 8.1. The image $\omega_{n}$ is nontrivial.}

The organization of the remaining sections is as follows: In Section 2 we review the results in [Torii 2010a]. We recall the construction of a commutative ring spectrum $\mathbb{B}$ which is an extension of both of $E_{n}$ and $E_{n+1}$, and the action of the group $\mathbb{G}=G_{n} \times_{\Gamma} G_{n+1}$ on $\mathbb{B}$. In Section 3 we introduce a topology for $\mathbb{A}^{*}$-modules of certain type, and study modules of continuous maps from a topological space to such a topological $\mathbb{A}^{*}$-module. In particular, we show that the functor $\operatorname{Map}_{c}\left(T, \mathbb{A}^{*}(-)\right)$ is a generalized cohomology theory for any compact space $T$. In Section 4 we construct the $K(n)$-localization of the $K(n+1)$-local $E_{n+1}$-Adams spectral sequence by applying the $K(n)$-localization functor to the $K(n+1)$-local $E_{n+1}$-Adams resolution of $L_{K(n+1)} S^{0}$, and prove Theorem 4.7. In Section 5 we define a cohomology of $\mathbb{G}$ with coefficients in $\mathbb{B}^{*}(W)$ for the purpose of connecting the cohomology of $G_{n}$ and that of $G_{n+1}$. Then we show that the inflation map from the cohomology of $G_{n+1}$ with coefficients in $\mathbb{A}^{*}(W)$ to the cohomology of $\mathbb{G}$ with coefficients in $\mathbb{B}^{*}(W)$ is an isomorphism for any spectrum $W$. In Section 6 we construct a map of spectral sequences from the $K(n)$-local $E_{n}$-Adams spectral sequence to the $K(n)$-localization of the $K(n+1)$-local $E_{n+1^{-}}$ Adams spectral sequence. In Section 7 we construct a homomorphism $\theta(W)$ from the cohomology group of $G_{n}$ with coefficients in $E_{n}^{*}(W)$ to the cohomology group of $G_{n+1}$ with coefficients in $\mathbb{A}^{*}(W)$ by using the cohomology of $\mathbb{G}$ with coefficients in $\mathbb{B}^{*}(W)$ constructed in Section 5. Then we identify this homomorphism with the map of spectral sequences on $E_{2}$-terms, and prove Theorem 7.6. In Section 8 we prove Theorem 8.1 as an application of the results obtained earlier.

\section{The ring spectrum $\mathbb{B}$}

In this section we review the results in [Torii 2010a]. We recall the construction of a commutative ring spectrum $\mathbb{B}$ and two ring spectrum maps $\Theta: E_{n+1} \rightarrow \mathbb{B}$ and $I: E_{n} \rightarrow \mathbb{B}$. Furthermore, we recall that the action of a profinite group $\mathbb{G}$ on $\mathbb{B}$ and the equivariance of $\Theta$ and $I$ under the actions of $\mathbb{G}$.

Let $p$ be a prime number, and let $n$ be a positive integer. We fix a finite field $\boldsymbol{F}$ which contains the finite fields $\mathbb{F}_{p^{n}}$ and $\mathbb{F}_{p^{n+1}}$. Note that the minimal field satisfying the condition is $\mathbb{F}_{p^{n}} \otimes \mathbb{F}_{p^{n+1}} \cong \mathbb{F}_{p^{n^{2}+n}}$. We denote by $W$ the ring of Witt vectors with coefficients in $\boldsymbol{F}$. We define variants of the $n$-th Morava $E$-theory spectrum $E_{n}$ and the $(n+1)$-st Morava $E$-theory spectrum $E_{n+1}$ such that the coefficient rings 
are given by

$$
E_{n}^{*}=W \llbracket w_{1}, \ldots, w_{n-1} \rrbracket\left[w^{ \pm 1}\right], \quad E_{n+1}^{*}=W \llbracket u_{1}, \ldots, u_{n} \rrbracket\left[u^{ \pm 1}\right] .
$$

There is an associated degree 0 formal group law $F_{n}$ over $E_{n}^{0}$ since $E_{n}$ is complex oriented and even-periodic. The formal group law $F_{n}$ is a universal deformation of the Honda formal group law $H_{n}$ of height $n$ over $\boldsymbol{F}$. Note that we can take $F_{n}$ as a $p$-typical formal group law. The Morava stabilizer group $S_{n}$ is defined to be the group of automorphisms of $H_{n}$ over $\boldsymbol{F}$. Then the extended Morava stabilizer group $G_{n}$ is defined to be the semi-direct product $G_{n}=\Gamma \ltimes S_{n}$, where $\Gamma=\operatorname{Gal}\left(\boldsymbol{F} / \mathbb{F}_{p}\right)$ is the Galois group of $\boldsymbol{F}$ over the prime field $\mathbb{F}_{p}$. We can identify $G_{n}$ with the group of automorphisms of the ring spectrum $E_{n}$ in the stable homotopy category. Then $g=(\gamma, s) \in \Gamma \ltimes S_{n}=G_{n}$ induces a ring homomorphism $g^{*}: E_{n}^{*} \rightarrow E_{n}^{*}$. We denote by $F_{n}^{g}$ the formal group law obtained from $F_{n}$ by the coefficient change along $g^{*}$. Then there is a unique isomorphism $t(g): F_{n} \rightarrow F_{n}^{g}$ of formal group laws which is a lifting of the isomorphism $s: H_{n} \rightarrow H_{n}^{\gamma}=H_{n}$. There are projections $G_{n} \rightarrow \Gamma$ and $G_{n+1} \rightarrow \Gamma$. We define a profinite group $\mathbb{G}$ to be the fiber product of $G_{n}$ and $G_{n+1}$ over $\Gamma$

$$
\mathbb{G}=G_{n} \times_{\Gamma} G_{n+1} .
$$

Let $K(n)$ be the $n$-th Morava $K$-theory spectrum at $p$. We denote by $\mathbb{A}$ the commutative ring spectrum $L_{K(n)} E_{n+1}$, the Bousfield localization of $E_{n+1}$ with respect to $K(n)$. The coefficient ring of $\mathbb{A}$ is given by the following Lemma.

Lemma 2.1. The coefficient ring $\mathbb{A}^{*}$ is isomorphic to $\left(E_{n+1}^{*}\left[u_{n}^{-1}\right]\right)_{I_{n}}^{\wedge}$, the completion of the localization $E_{n+1}^{*}\left[u_{n}^{-1}\right]$ at the ideal $I_{n}=\left(p, u_{1}, \ldots, u_{n-1}\right)$. Hence $\mathbb{A}^{*}$ is a graded complete Noetherian regular local ring isomorphic to

$$
\left(W\left(\left(u_{n}\right)\right)\right)_{p}^{\wedge} \llbracket u_{1}, \ldots, u_{n-1} \rrbracket\left[u^{ \pm 1}\right]
$$

with residue field $\boldsymbol{F}\left(\left(u_{n}\right)\right)\left[u^{ \pm 1}\right]$.

Proof. There is a tower $\{M(J)\}_{J}$ of generalized Moore spectra of height $n$ as in [Hovey and Strickland 1999, Proposition 4.2]. If $J=\left(p^{a_{0}}, v_{1}^{a_{1}}, \ldots, v_{n-1}^{a_{n-1}}\right)$, then $\left(E_{n+1} \wedge M(J)\right)^{*}=E_{n+1}^{*} /\left(p^{a_{0}}, u_{1}^{a_{1}}, \ldots, u_{n-1}^{a_{n-1}}\right)$ since $v_{i}=u_{i} u^{p^{i}-1}$ for $i=$ $1, \ldots, n-1$. We set $X_{I_{n}}^{\wedge}=\operatorname{holim}_{J} X \wedge M(J)$ for a spectrum $X$. Since $E_{n+1}$ is Landweber exact of height $(n+1)$, it satisfies the telescope conjecture at $n$ in the sense of [Hovey 1997, Definition 1.5.2]. Then $L_{K(n)} E_{n+1} \simeq\left(E_{n+1}\left[v^{-1}\right]\right)_{I_{n}}^{\wedge}$ by [Hovey 1997, Theorem 1.5.4], where $v$ is a generalized $v_{n}$-element in $E_{n+1}^{*}$ in the sense of [Hovey 1997, Definition 1.2.2]. We can take $v_{n}=u_{n} u^{p^{n}-1} \in \pi_{2 p^{n}-2} E_{n+1}$ as a generalized $v_{n}$-element. Since the sequence $p^{a_{0}}, u_{1}^{a_{1}}, \ldots, u_{n-1}^{a_{n-1}}$ is regular in $E_{n+1}^{*}\left[v_{n}^{-1}\right]=E_{n+1}^{*}\left[u_{n}^{-1}\right],\left(E_{n+1}\left[v_{n}^{-1}\right] \wedge M(J)\right)^{*}=E_{n+1}^{*}\left[u_{n}^{-1}\right] /\left(p^{a_{0}}, u_{1}^{a_{1}}, \ldots, u_{n-1}^{a_{n-1}}\right)$ if $J=\left(p^{a_{0}}, v_{1}^{a_{1}}, \ldots, v_{n-1}^{a_{n-1}}\right)$. Then we see that $\mathbb{A}^{*}=\left(L_{K(n)} E_{n+1}\right)^{*}$ is the completion of $E_{n+1}^{*}\left[u_{n}^{-1}\right]$ at the ideal $I_{n}=\left(p, u_{1}, \ldots, u_{n-1}\right): \mathbb{A}^{*} \cong\left(E_{n+1}^{*}\left[u_{n}^{ \pm 1}\right]\right)_{I_{n}}^{\wedge}$. Since the 
sequence $p, u_{1}, \ldots, u_{n-1}$ is regular in $E_{n+1}^{*}\left[u_{n}^{ \pm 1}\right]$, and it generates a maximal ideal, $\mathbb{A}^{*}$ is a graded regular local ring with maximal ideal generated by $p, u_{1}, \ldots, u_{n-1}$ and residue field $\boldsymbol{F}\left(\left(u_{n}\right)\right)\left[u^{ \pm 1}\right]$.

The obvious ring homomorphism $W \llbracket u_{n} \rrbracket \rightarrow \mathbb{A}^{*}$ extends to $\left(W\left(\left(u_{n}\right)\right)\right)_{p}^{\wedge} \rightarrow \mathbb{A}^{*}$, since $u_{n}$ is a unit in $\mathbb{A}^{*}$, and $\mathbb{A}^{*}$ is $p$-complete. Furthermore, since $\mathbb{A}^{*}$ is $I_{n}$-adically complete, the obvious ring homomorphism $\left(W\left(\left(u_{n}\right)\right)\right)_{p}^{\wedge}\left[u_{1}, \ldots, u_{n-1}\right]\left[u^{ \pm 1}\right] \rightarrow \mathbb{A}^{*}$ extends to $\left(W\left(\left(u_{n}\right)\right)\right){ }_{p}^{\wedge} \llbracket u_{1}, \ldots, u_{n-1} \rrbracket\left[u^{ \pm 1}\right] \rightarrow \mathbb{A}^{*}$. The ring

$$
\left(W\left(\left(u_{n}\right)\right)\right)_{p}^{\wedge} \llbracket u_{1}, \ldots, u_{n-1} \rrbracket\left[u^{ \pm 1}\right]
$$

is a graded complete regular local ring with maximal ideal generated by $p, u_{1}, \ldots$, $u_{n-1}$ and residue field $\boldsymbol{F}\left(\left(u_{n}\right)\right)\left[u^{ \pm 1}\right]$. Since the ring homomorphism

$$
\left(W\left(\left(u_{n}\right)\right)\right)_{p}^{\wedge} \llbracket u_{1}, \ldots, u_{n-1} \rrbracket\left[u^{ \pm 1}\right] \rightarrow \mathbb{A}^{*}
$$

is continuous, and it induces an isomorphism on the associated graded rings, we obtain an isomorphism between $\mathbb{A}^{*}$ and $\left(W\left(\left(u_{n}\right)\right)\right)_{p}^{\wedge} \llbracket u_{1}, \ldots, u_{n-1} \rrbracket\left[u^{ \pm 1}\right]$.

Since a complete local ring is Henselian, $\mathbb{A}^{*}$ is a Henselian ring by Lemma 2.1.

Lemma 2.2 [Milne 1980, Proposition I.4.4]. Let $R$ be a Henselian ring with residue field $k$. Then the functor $S \mapsto S \otimes_{R} k$ induces an equivalence between the category of finite étale $R$-algebras and the category of finite étale $k$-algebras.

Let $\bar{F}_{n+1}$ be the formal group law over $\boldsymbol{F}\left(\left(u_{n}\right)\right)$ obtained from $F_{n+1}$ by the reduction $E_{n+1}^{0} \rightarrow \boldsymbol{F}\left(\left(u_{n}\right)\right)$. Then the height of $\bar{F}_{n+1}$ is $n$. Since the isomorphism classes of formal group laws over a separably closed field are classified by their height, there is an isomorphism between $\bar{F}_{n+1}$ and the height $n$ Honda formal group law $H_{n}$ over the separable closure $\boldsymbol{F}\left(\left(u_{n}\right)\right)^{\text {sep }}$. In [Torii 2003, §2.3] we have constructed an extension field $L$ of $\boldsymbol{F}\left(\left(u_{n}\right)\right)$, where $L$ is the minimal extension such that there is an isomorphism between $\bar{F}_{n+1}$ and $H_{n}$. The extension $L$ is Galois over $\boldsymbol{F}\left(\left(u_{n}\right)\right)$ with Galois group isomorphic to $S_{n}$. There is a sequence of finite Galois extensions of $\boldsymbol{F}\left(\left(u_{n}\right)\right)$

$$
\boldsymbol{F}\left(\left(u_{n}\right)\right)=L(-1) \rightarrow L(0) \rightarrow L(1) \rightarrow \cdots
$$

such that $L=\bigcup_{i} L(i)$. We denote by $S_{n}(i)$ the Galois group for $\boldsymbol{F}\left(\left(u_{n}\right)\right) \rightarrow L(i)$. Then $S_{n}(i)$ is a finite quotient group of $S_{n}$ of order $\left(p^{n}-1\right) p^{n i}$, and $S_{n}=\lim _{i} S_{n}(i)$. The action of $G_{n+1}$ on $E_{n+1}^{0}$ induces an action on the residue field $\boldsymbol{F}\left(\left(u_{n}\right)\right)$ of $\mathbb{A}^{0}$. By [Torii 2003, §2.4], there is an action of $\mathbb{G}$ on $L$, which is an extension of the action of $G_{n+1}$ on $\boldsymbol{F}\left(\left(u_{n}\right)\right)$ and the action of $S_{n}$ on $L$ as Galois group. Note that $L(i)$ is stable under the action of $\mathbb{G}$ for all $i$. 
By Lemma 2.2, the sequence of Galois extensions (2-1) induces a sequence of graded commutative rings

$$
\mathbb{A}^{*}=\mathbb{B}(-1)^{*} \rightarrow \mathbb{B}(0)^{*} \rightarrow \mathbb{B}(1)^{*} \rightarrow \cdots .
$$

The ring $\mathbb{B}(i)^{*}$ is an even-periodic graded complete Noetherian regular local ring with residue field $L(i)\left[u^{ \pm 1}\right]$. Furthermore, $\mathbb{A}^{*} \rightarrow \mathbb{B}(i)^{*}$ is a Galois extension of graded commutative rings with Galois group $S_{n}(i)$ in the sense of [Chase et al. 1965; Greither 1992]. Let $\mathbb{B}(\infty)^{*}$ be the direct limit of the sequence: $\mathbb{B}(\infty)^{*}=$ colim $_{i} \mathbb{B}(i)^{*}$. Then we define a graded commutative ring $\mathbb{B}^{*}$ to be the completion of $\mathbb{B}(\infty)^{*}$ at the ideal $I_{n}=\left(p, u_{1}, \ldots, u_{n-1}\right)$

$$
\mathbb{B}^{*}=\left(\mathbb{B}(\infty)^{*}\right)_{I_{n}}^{\wedge} .
$$

By Lemma 2.2, there is a unique lifting of the action of $\mathbb{G}$ on $\mathbb{B}^{*}$ and $\mathbb{B}(i)^{*}$ for $0 \leq i \leq \infty$ compatible with canonical inclusions.

By the $\mathbb{A}^{*}$-algebra structures, we can regard $\mathbb{B}^{*}$ and $\mathbb{B}(i)^{*}$ for $0 \leq i \leq \infty$ as Landweber exact even-periodic graded commutative rings. We denote the corresponding commutative ring spectra by $\mathbb{B}$ and $\mathbb{B}(i)$ for $0 \leq i \leq \infty$, respectively. Hence we obtain a sequence of commutative ring spectra

$$
\mathbb{A}=\mathbb{B}(-1) \rightarrow \mathbb{B}(0) \rightarrow \mathbb{B}(1) \rightarrow \cdots .
$$

Then we have $\mathbb{B}(\infty)=$ hocolim $_{i} \mathbb{B}(i)$ and $\mathbb{B}=L_{K(n)} \mathbb{B}(\infty)$. We define a ring spectrum map $\Theta: E_{n+1} \rightarrow \mathbb{B}$ to be the composition

$$
\Theta: E_{n+1} \longrightarrow L_{K(n)} E_{n+1}=\mathbb{A} \longrightarrow \mathbb{B} .
$$

By [Torii 2003, §2.3], the formal group law induced by the ring homomorphism $E_{n}^{0} \rightarrow \boldsymbol{F} \hookrightarrow L$ is isomorphic to the formal group law induced by the ring homomorphism $E_{n+1}^{0} \rightarrow \boldsymbol{F}\left(\left(u_{n}\right)\right) \hookrightarrow L$. By the universality of the formal group law $F_{n}$ associated with $E_{n}$, there exists a ring homomorphism $E_{n}^{*} \rightarrow \mathbb{B}^{*}$ and an isomorphism $\Phi$ between the formal group laws $F_{n}$ and $F_{n+1}$ over $\mathbb{B}^{0}$

$$
\Phi: F_{n+1} \stackrel{\cong}{\longrightarrow} F_{n} \text {. }
$$

Note that $\mathbb{B}^{0}$ is the minimal extension ring of both of $E_{n}^{0}$ and $E_{n+1}^{0}$ such that there exists an isomorphism between $F_{n}$ and $F_{n+1}$. Since $E_{n}$ and $\mathbb{B}$ are even-periodic Landweber exact commutative ring spectra, the ring homomorphism $E_{n}^{*} \rightarrow \mathbb{B}^{*}$ extends to a ring spectrum map

$$
I: E_{n} \longrightarrow \mathbb{B} .
$$

By the projection $\mathbb{G} \rightarrow G_{n}$, we can consider that $\mathbb{G}$ acts on $E_{n}$ as automorphisms of commutative ring spectrum in the stable homotopy category. Also, by 
the projection $\mathbb{G} \rightarrow G_{n+1}$, we can consider that $\mathbb{G}$ acts on $E_{n+1}$ as automorphisms of commutative ring spectrum.

Proposition 2.3 [Torii 2010a, §4]. The profinite group $\mathbb{G}$ acts on the commutative ring spectrum $\mathbb{B}$ in the stable homotopy category. The ring spectrum maps $I$ : $E_{n} \rightarrow \mathbb{B}$ and $\Theta: E_{n+1} \rightarrow \mathbb{B}$ are equivariant with respect to the actions of $\mathbb{G}$.

Remark 2.4 [Torii 2010b]. The ring spectrum $\mathbb{B}$ supports a commutative $S$-algebra structure and the group $\mathbb{G}$ acts on $\mathbb{B}$ in the category of commutative $S$-algebras. Let $T=L_{K(n)} S^{0} \otimes_{\mathbb{Z}_{p}} W$ be the commutative $S$-algebra obtained from $L_{K(n)} S^{0}$ by adjoining a primitive $\left(p^{m}-1\right)$-st root of unity, where $m$ is the dimension of $\boldsymbol{F}$ over $\mathbb{F}_{p}$. Then there is an equivalence $\mathbb{B} \simeq L_{K(n)}\left(E_{n} \wedge{ }_{T} \mathbb{A}\right)$ of commutative $S$-algebras. In particular, when $\boldsymbol{F}=\mathbb{F}_{p^{n^{2}+n}}$, there is an equivalence $\mathbb{B} \simeq L_{K(n)}\left(E_{n}^{\prime} \wedge E_{n+1}^{\prime}\right)$ of commutative $S$-algebras, where $E_{n}^{\prime}$ and $E_{n+1}^{\prime}$ are the standard Morava $E$-theory spectra so that $\pi_{0} E_{n}^{\prime} / I_{n}=\mathbb{F}_{p^{n}}$ and $\pi_{0} E_{n+1}^{\prime} / I_{n+1}=\mathbb{F}_{p^{n+1}}$. In this case

$$
\operatorname{Gal}\left(\boldsymbol{F} / \mathbb{F}_{p}\right) \cong \operatorname{Gal}\left(\mathbb{F}_{p^{n}} / \mathbb{F}_{p}\right) \times \operatorname{Gal}\left(\mathbb{F}_{p^{n+1}} / \mathbb{F}_{p}\right) \quad \text { and } \quad \mathbb{G} \cong G_{n}^{\prime} \times G_{n+1}^{\prime},
$$

where $G_{n}^{\prime}=\operatorname{Gal}\left(\mathbb{F}_{p^{n}} / \mathbb{F}_{p}\right) \ltimes S_{n}$ and $G_{n+1}^{\prime}=\operatorname{Gal}\left(\mathbb{F}_{p^{n+1}} / \mathbb{F}_{p}\right) \ltimes S_{n+1}$ are the standard extended Morava stabilizer groups.

\section{Mapping space $\operatorname{Map}_{c}\left(T, \mathbb{A}^{*}(W)\right)$}

To interpret the $E_{2}$-term of the $K(n)$-localization of the $K(n+1)$-local $E_{n+1^{-}}$ Adams spectral sequence which will be constructed in Section 4 below as a cohomology group of $G_{n+1}$, we need to give an appropriate topology for $\mathbb{A}^{*}$-cohomology groups. In this section we introduce a topology for $\mathbb{A}^{*}$-modules of certain type, and study modules of continuous maps from a topological space to such an $\mathbb{A}^{*}$-module.

For a topological space $T$, and a topological module $M$, denote by $\operatorname{Map}_{c}(T, M)$ the module of continuous maps from $T$ to $M$. Recall the fact that a surjection between profinite groups has a continuous section of topological spaces [Serre 1994, Proposition I.1.2.1]. This implies that $\operatorname{Map}_{c}(T,-)$ gives an exact functor from the category of profinite modules to that of abelian groups. The coefficient ring $E_{n+1}^{*}$ is a graded complete Noetherian local ring with maximal ideal $I_{n+1}=$ $\left(p, u_{1}, \ldots, u_{n}\right)$. Since $E_{n+1}^{*} / I_{n+1}^{r}$ is a graded finite ring for each $r, E_{n+1}^{*}$ is a graded profinite ring. Let $N$ be a finitely generated $E_{n+1}^{*}$-module. Then $N$ is a graded profinite abelian group. In this case there is an easy description for $\operatorname{Map}_{c}(T, N)$ as follows.

Lemma 3.1. If $N$ is a finitely generated $E_{n+1}^{*}$-module, there is a natural isomorphism

$$
\operatorname{Map}_{c}(T, N) \cong \operatorname{Map}_{c}\left(T, E_{n+1}^{*}\right) \otimes_{E_{n+1}^{*}} N
$$


Proof. Since $N$ is finitely generated, there is an exact sequence of profinite modules $N^{1} \rightarrow N^{0} \rightarrow N \rightarrow 0$, where $N^{i}$ is finitely generated free for $i=0,1$. This induces two exact sequences $\operatorname{Map}_{c}\left(T, N^{1}\right) \rightarrow \operatorname{Map}_{c}\left(T, N^{0}\right) \rightarrow \operatorname{Map}_{c}(T, N) \rightarrow 0$ and $\operatorname{Map}_{c}\left(T, E_{n+1}^{*}\right) \otimes N^{1} \rightarrow \operatorname{Map}_{c}\left(T, E_{n+1}^{*}\right) \otimes N^{0} \rightarrow \operatorname{Map}_{c}\left(T, E_{n+1}^{*}\right) \otimes N \rightarrow 0$. Since $N^{i}$ is finitely generated free, we have $\operatorname{Map}_{c}\left(T, N^{i}\right) \cong \operatorname{Map}_{c}\left(T, E_{n+1}^{*}\right) \otimes N^{i}$ for $i=0,1$. Hence we obtain that $\operatorname{Map}_{c}(T, N) \cong \operatorname{Map}_{c}\left(T, E_{n+1}^{*}\right) \otimes N$.

Corollary 3.2. For an ideal I of $E_{n+1}^{*}$ and a finitely generated $E_{n+1}^{*}$-module $N$, there is a natural isomorphism

$$
\operatorname{Map}_{c}(T, N / I N) \cong \operatorname{Map}_{c}(T, N) / I \operatorname{Map}_{c}(T, N) .
$$

By Lemma 3.1, it is fundamental to understand $\operatorname{Map}_{c}\left(T, E_{n+1}^{*}\right)$. Recall that a module over a (graded) regular local ring is called profree if it is isomorphic to the completion at the maximal ideal of some free module (see [Hovey and Strickland 1999, Theorem A.9] for equivalent conditions of profree modules).

Proposition 3.3. For a topological space $T, \operatorname{Map}_{c}\left(T, E_{n+1}^{*}\right)$ is a profree $E_{n+1}^{*}$ module.

Proof. Put $P=\operatorname{Map}_{c}\left(T, E_{n+1}^{*}\right)$. We have $P \cong \lim _{r} \operatorname{Map}_{c}\left(T, E_{n+1}^{*} / I_{n+1}^{r}\right)$, since $E_{n+1}^{*} \cong \lim _{r} E_{n+1}^{*} / I_{n+1}^{r}$. Then $P \cong \lim _{r} P / I_{n+1}^{r} P$ by Corollary 3.2. This shows that $P$ is $L$-complete by [Hovey and Strickland 1999, Theorem A.6(a)]. Since $p, u_{1}, \ldots, u_{n}$ is a regular sequence on $E_{n+1}^{*}$,

$$
0 \rightarrow E_{n+1}^{*} / I_{k} \stackrel{u_{k}}{\rightarrow} E_{n+1}^{*} / I_{k} \rightarrow E_{n+1}^{*} / I_{k+1} \rightarrow 0
$$

is an exact sequence of profinite modules for $k=0,1, \ldots, n$. By applying the functor $\operatorname{Map}_{c}(T,-)$, we obtain an exact sequence

$$
0 \rightarrow P / I_{k} P \stackrel{u_{k}}{\rightarrow} P / I_{k} P \rightarrow P / I_{k+1} P \rightarrow 0
$$

for $k=0,1, \ldots, n$ by Corollary 3.2. Hence $p, u_{1}, \ldots, u_{n}$ is a regular sequence on $P$, and $P$ is profree by [Hovey and Strickland 1999, Theorem A.9].

Recall that $\mathbb{A}=L_{K(n)} E_{n+1}$ and $\mathbb{A}^{*} \cong E_{n+1}^{*}\left[u_{n}^{-1}\right]_{I_{n}}^{\wedge}=\lim _{r} E_{n+1}^{*} / I_{n}^{r}\left[u_{n}^{-1}\right]$ by Lemma 2.1. We denote by $J_{n}$ the ideal of $\mathbb{A}^{*}$ generated by $p, u_{1}, \ldots, u_{n-1}$, that is, $J_{n}=I_{n} \mathbb{A}^{*} \subset \mathbb{A}^{*}$. Then we have $\mathbb{A}^{*} / J_{n}^{r}=E_{n+1}^{*} / I_{n}^{r}\left[u_{n}^{-1}\right]$. Note that $\mathbb{A}^{*} / J_{n}^{r}$ is a graded ring of formal Laurent series over an Artinian local ring. To introduce a topology for $\mathbb{A}^{*}$-modules of certain type, we first consider the case of such a ring.

Definition 3.4. Let $R$ be a (graded) Artinian local ring. Then the ring $R \llbracket a \rrbracket$ of formal power series is a Noetherian local ring. Note that the topology of $R \llbracket a \rrbracket$ coincides with the $(a)$-adic topology since the maximal ideal of $R$ is nilpotent. We give the ring $R((a))=R \llbracket a \rrbracket\left[a^{-1}\right]$ of formal Laurent series a $R \llbracket a \rrbracket$-linear topology such that $R \llbracket a \rrbracket$ is an open submodule. Then $R((a))$ is a union of open submodules 
$a^{r} R \llbracket a \rrbracket$ for $r \in \mathbb{Z}: R((a))=\bigcup_{r \in \mathbb{Z}} a^{r} R \llbracket a \rrbracket$. For an $R \llbracket a \rrbracket$-module $N$, we give the (a)-adic topology on $N$. The localization $N\left[a^{-1}\right]$ is an $R((a))$-module. Let $N^{\prime}$ be the image of the localization map $N \rightarrow N\left[a^{-1}\right]$. Then $N^{\prime}$ is an $R \llbracket a \rrbracket-$ submodule of $N\left[a^{-1}\right]$. We give an $R \llbracket a \rrbracket$-linear topology on $N\left[a^{-1}\right]$ such that $N^{\prime}$ is an open submodule. Then $N\left[a^{-1}\right]$ is a union of open submodules $a^{r} N^{\prime}$ for $r \in \mathbb{Z}$ : $N\left[a^{-1}\right]=\bigcup_{r \in \mathbb{Z}} a^{r} N^{\prime}$.

For an $R \llbracket a \rrbracket$-module $N$, the localization map $N \rightarrow N\left[a^{-1}\right]$ induces a map $\operatorname{Map}_{c}(T, N)\left[a^{-1}\right] \rightarrow \operatorname{Map}_{c}\left(T, N\left[a^{-1}\right]\right)$ of $R((a))$-modules. The following lemma gives a sufficient condition that this map is an isomorphism.

Lemma 3.5. Let $R$ be a (graded) Artinian local ring with finite residue field, and let $T$ be a compact space. For an $R \llbracket a \rrbracket$-module $N$, there is a natural isomorphism

$$
\operatorname{Map}_{c}\left(T, N\left[a^{-1}\right]\right) \cong \operatorname{Map}_{c}\left(T, N^{\prime}\right)\left[a^{-1}\right],
$$

where $N^{\prime}$ is the image of the localization map $N \rightarrow N\left[a^{-1}\right]$. Furthermore, if $N$ is (a)-torsion free or finitely generated, then there is a natural isomorphism

$$
\operatorname{Map}_{c}\left(T, N\left[a^{-1}\right]\right) \cong \operatorname{Map}_{c}(T, N)\left[a^{-1}\right] .
$$

Proof. Since $N\left[a^{-1}\right]$ is a union of open submodules $a^{r} N^{\prime}$ for $r \in \mathbb{Z}$, any continuous map from $T$ to $N\left[a^{-1}\right]$ factors through $a^{r} N^{\prime}$ for some $r$. Hence

$$
\operatorname{Map}_{c}\left(T, N^{\prime}\right)\left[a^{-1}\right] \stackrel{\cong}{\rightarrow} \operatorname{Map}_{c}\left(T, N\left[a^{-1}\right]\right) .
$$

If $N$ is $(a)$-torsion free, then $N^{\prime}=N$. Assume that $N$ is finitely generated. Let $K$ be the kernel of the surjection $N \rightarrow N^{\prime}$. Since $N\left[a^{-1}\right] \cong N^{\prime}\left[a^{-1}\right], K\left[a^{-1}\right]=0$. Since $K$ is finitely generated, there is a positive integer $m$ such that $a^{m} K=0$. Since $R \llbracket a \rrbracket$ is profinite, $\operatorname{Map}_{c}(T,-)$ is an exact functor on the category of finitely generated $R \llbracket a \rrbracket$-modules. Then the exact sequence $0 \rightarrow K \rightarrow N \rightarrow N^{\prime} \rightarrow 0$ induces an exact sequence $0 \rightarrow \operatorname{Map}_{c}(T, K) \rightarrow \operatorname{Map}_{c}(T, N) \rightarrow \operatorname{Map}_{c}\left(T, N^{\prime}\right) \rightarrow 0$. The fact that $a^{m} K=0$ implies $a^{m} \operatorname{Map}_{c}(T, K)=0$. Hence $\operatorname{Map}_{c}(T, K)\left[a^{-1}\right]=0$. So we obtain that $\operatorname{Map}_{c}(T, N)\left[a^{-1}\right] \cong \operatorname{Map}_{c}\left(T, N^{\prime}\right)\left[a^{-1}\right]$.

We define a topology for $\mathbb{A}^{*}$-modules of the form $\lim _{r} N / I_{n}^{r}\left[u_{n}^{-1}\right]$ for some $E_{n+1}^{*}$-module $N$.

Definition 3.6. For an $\mathbb{A}^{*} / J_{n}^{r}$-module $M$, since $\mathbb{A}^{*} / J_{n}^{r}$ is a graded ring of formal Laurent series over an Artinian local ring, we give a topology on $M$ as in Definition 3.4. For an $E_{n+1}^{*}$-module $N$, we define an $\mathbb{A}^{*}$-module $\mathbb{A}^{*} N$ by

$$
\mathbb{A}^{*} N=N\left[u_{n}^{-1}\right]_{I_{n}}^{\wedge}=\lim _{r} N / I_{n}^{r} N\left[u_{n}^{-1}\right] .
$$

Then $N / I_{n}^{r}\left[u_{n}^{-1}\right]$ is an $\mathbb{A}^{*} / J_{n}^{r}$-module. We give $\mathbb{A}^{*} N=\lim _{r} N / I_{n}^{r} N\left[u_{n}^{-1}\right]$ a topology by using the inverse limit topology. 
Note that there is an isomorphism $\mathbb{A}^{*} E_{n+1}^{*} \cong \mathbb{A}^{*}$. If $N$ is a finitely generated $E_{n+1}^{*}$-module, then $N\left[u_{n}^{-1}\right]$ is finitely generated over the Noetherian ring $E_{n+1}^{*}\left[u_{n}^{-1}\right]$. Then the completion of $N\left[u_{n}^{-1}\right]$ at the ideal $I_{n}$ is given by the tensor product with $\mathbb{A}^{*}$. Hence there is a natural isomorphism $\mathbb{A}^{*} N \cong \mathbb{A}^{*} \otimes_{E_{n+1}^{*}} N$ for any finitely generated $E_{n+1}^{*}$-module $N$, and the functor $\mathbb{A}^{*}(-)$ is exact on the category of finitely generated $E_{n+1}^{*}$-modules.

In the rest of this section we study the functor $\operatorname{Map}_{c}\left(T, \mathbb{A}^{*}(-)\right)$ with $T$ compact.

Lemma 3.7. If $T$ is a compact space and $N$ is a finitely generated $E_{n+1}^{*}$-module, then there is a natural isomorphism of $\mathbb{A}^{*}$-modules

$$
\operatorname{Map}_{c}\left(T, \mathbb{A}^{*} N\right) \cong \mathbb{A}^{*} \operatorname{Map}_{c}(T, N) .
$$

Proof. Since $\mathbb{A}^{*} N=\lim _{r} N / I_{n}^{r} N\left[u_{n}^{-1}\right]$, we have

$$
\operatorname{Map}_{c}\left(T, \mathbb{A}^{*} N\right) \cong \lim _{r} \operatorname{Map}_{c}\left(T, N / I_{n}^{r} N\left[u_{n}^{-1}\right]\right) \text {. }
$$

By Lemma 3.5 and Corollary 3.2,

$$
\operatorname{Map}_{c}\left(T, N / I_{n}^{r} N\left[u_{n}^{-1}\right]\right) \cong \operatorname{Map}_{c}(T, N) / I_{n}^{r} \operatorname{Map}_{c}(T, N)\left[u_{n}^{-1}\right] .
$$

Hence $\operatorname{Map}_{c}\left(T, \mathbb{A}^{*} N\right)$ is isomorphic to $\lim _{r} \operatorname{Map}_{c}(T, N) / I_{n}^{r} \operatorname{Map}_{c}(T, N)\left[u_{n}^{-1}\right]=$ $\mathbb{A}^{*} \operatorname{Map}_{c}(T, N)$.

The basic case is when $N=E_{n+1}^{*}$ :

Proposition 3.8. For any compact space $T, \operatorname{Map}_{c}\left(T, \mathbb{A}^{*}\right)$ is a profree $\mathbb{A}^{*}$-module.

Proof. By Proposition 3.3, $\operatorname{Map}_{c}\left(T, E_{n+1}^{*}\right)$ is profree over $E_{n+1}^{*}$, and is thus a direct summand of some product $\prod_{\alpha} E_{n+1}^{*}$ by [Hovey and Strickland 1999, Proposition A.13]. Hence it is sufficient to show that $\mathbb{A}^{*}\left(\prod_{\alpha} E_{n+1}^{*}\right)$ is profree over $\mathbb{A}^{*}$. For $k=0,1, \ldots, n-1$, we put $M=E_{n+1}^{*} / I_{k}$ and $N=E_{n+1}^{*} / I_{k+1}$. Let $K_{r}$ be the kernel of the map $M / I_{n}^{r} M \stackrel{u_{k}}{\rightarrow} M / I_{n}^{r} M$, and let $L_{r}$ be the kernel of the map $M / I_{n}^{r} M \rightarrow N / I_{n}^{r} N$. Then there are exact sequences $0 \rightarrow K_{r} \rightarrow M / I_{n}^{r} M \rightarrow L_{r} \rightarrow 0$ and $0 \rightarrow L_{r} \rightarrow M / I_{n}^{r} M \rightarrow N / I_{n}^{r} N \rightarrow 0$. Since $E_{n+1}^{*}$ is regular, the canonical map $K_{r+1} \rightarrow K_{r}$ is 0 . Then

$$
\lim _{r}\left(\left(\prod_{\alpha} K_{r}\right)\left[u_{n}^{-1}\right]\right)=\lim _{r}^{1}\left(\left(\prod_{\alpha} K_{r}\right)\left[u_{n}^{-1}\right]\right)=0 .
$$

Hence we obtain $\lim _{r}\left(\left(\prod_{\alpha} M / I_{n}^{r} M\right)\left[u_{n}^{-1}\right]\right) \stackrel{\cong}{\rightrightarrows} \lim _{r}\left(\left(\prod_{\alpha} L_{r}\right)\left[u_{n}^{-1}\right]\right)$, and

$$
0=\lim _{r}^{1}\left(\left(\prod_{\alpha} M / I_{n}^{r} M\right)\left[u_{n}^{-1}\right]\right) \cong \lim _{r}^{1}\left(\left(\prod_{\alpha} L_{r}\right)\left[u_{n}^{-1}\right]\right) .
$$

This implies that the sequence

$$
\begin{aligned}
0 \rightarrow \lim _{r}\left(\left(\prod_{\alpha} M / I_{n}^{r}\right)\left[u_{n}^{-1}\right]\right) & \stackrel{u_{k}}{\longrightarrow} \lim _{r}\left(\left(\prod_{\alpha} M / I_{n}^{r} M\right)\left[u_{n}^{-1}\right]\right) \longrightarrow{\underset{\lim }{r}}_{r}\left(\left(\prod_{\alpha} N / I_{n}^{r} N\right)\left[u_{n}^{-1}\right]\right) \rightarrow 0
\end{aligned}
$$


is exact. This shows that $p, u_{1}, \ldots, u_{n-1}$ is a regular sequence on $\mathbb{A}^{*}\left(\prod_{\alpha} E_{n+1}^{*}\right)$. Therefore $\mathbb{A}^{*}\left(\prod_{\alpha} E_{n+1}^{*}\right)$ is profree $\mathbb{A}^{*}$-module by [Hovey and Strickland 1999, Theorem A.9].

The map from $T$ to the one point space $*$ induces a ring homomorphism $\mathbb{A}^{*}=$ $\operatorname{Map}_{c}\left(*, \mathbb{A}^{*}\right) \rightarrow \operatorname{Map}_{c}\left(T, \mathbb{A}^{*}\right)$. Then the composition with the commutative $M U^{*}$ algebra structure map $M U^{*} \rightarrow \mathbb{A}^{*}$ gives $\operatorname{Map}_{c}\left(T, \mathbb{A}^{*}\right)$ a commutative $M U^{*}$-algebra structure. Since a profree module over $\mathbb{A}^{*}$ is Landweber exact, we obtain the following corollary

Corollary 3.9. If $T$ is a compact space, then $\operatorname{Map}_{c}\left(T, \mathbb{A}^{*}\right)$ is Landweber exact.

We have a similar description for $\operatorname{Map}_{c}\left(T, \mathbb{A}^{*} N\right)$ as in Lemma 3.1 when $T$ is a compact space and $N$ is a finitely generated $E_{n+1}^{*}$-module as follows.

Proposition 3.10. If $T$ is a compact space and $N$ is a finitely generated $E_{n+1}^{*}$ module, then there is a natural isomorphism of $\mathbb{A}^{*}$-modules

$$
\operatorname{Map}_{c}\left(T, \mathbb{A}^{*} N\right) \cong \operatorname{Map}_{c}\left(T, \mathbb{A}^{*}\right) \underset{\mathbb{A}^{*}}{\otimes} \mathbb{A}^{*} N .
$$

For the proof of Proposition 3.10, we prepare the following (well-known) lemmas.

Lemma 3.11 ([Lam 1999, Proposition 4.4]). Let $R$ be a (graded) ring. If $M$ is a finitely presented module over $R$, then $\left(\prod_{\alpha} R\right) \otimes_{R} M \cong \prod_{\alpha} M$.

Proof. Since $M$ is finitely presented, there is an exact sequence $M^{1} \rightarrow M^{0} \rightarrow$ $M \rightarrow 0$, where $M^{i}$ is finitely generated free for $i=0,1$. Then there are two exact sequences $\left(\prod_{\alpha} R\right) \otimes M^{1} \rightarrow\left(\prod_{\alpha} R\right) \otimes M^{0} \rightarrow\left(\prod_{\alpha} R\right) \otimes M \rightarrow 0$ and $\prod_{\alpha} M^{1} \rightarrow$ $\prod_{\alpha} M^{0} \rightarrow \prod_{\alpha} M \rightarrow 0$. Since $M^{i}$ is finitely generated free, $\left(\prod_{\alpha} R\right) \otimes M^{i} \cong \prod_{\alpha} M^{i}$ for $i=0,1$. Hence we obtain $\left(\prod_{\alpha} R\right) \otimes M \cong \prod_{\alpha} M$.

Lemma 3.12. If $F$ is a profree $\mathbb{A}^{*}$-module and $M$ is a finitely generated $\mathbb{A}^{*}$ module, then $F \otimes_{\mathbb{A}^{*}} M$ is $J_{n^{-} \text {-adically complete. }}$

Proof. Since $F$ is profree, it is a direct summand of some product $\prod_{\alpha} \mathbb{A}^{*}$ by [Hovey and Strickland 1999, Proposition A.13]. Since a direct summand of complete module is complete, it is sufficient to show that $\left(\prod_{\alpha} \mathbb{A}^{*}\right) \otimes M$ is complete. By Lemma 3.11, $\left(\prod_{\alpha} \mathbb{A}^{*}\right) \otimes M \cong \prod_{\alpha} M$, and $\prod_{\alpha} M$ is complete.

Proof of Proposition 3.10. By Lemma 3.1, $\operatorname{Map}_{c}(T, N) \cong \operatorname{Map}_{c}\left(T, E_{n+1}^{*}\right) \otimes_{E_{n+1}^{*}} N$. Then we see that $\mathbb{A}^{*} \operatorname{Map}_{c}(T, N)$ is the completion of $\mathbb{A}^{*} \operatorname{Map}_{c}\left(T, E_{n+1}^{*}\right) \otimes_{\mathbb{A}^{*}} \mathbb{A}^{*} N$ at the ideal $J_{n}$. By Lemma 3.12, we see that $\mathbb{A}^{*} \operatorname{Map}_{c}\left(T, E_{n+1}^{*}\right) \otimes_{\mathbb{A}^{*}} \mathbb{A}^{*} N$ is $J_{n^{-}}$ adically complete. Hence we obtain

$$
\mathbb{A}^{*} \operatorname{Map}_{c}(T, N) \cong \mathbb{A}^{*} \operatorname{Map}_{c}\left(T, E_{n+1}^{*}\right) \otimes_{\mathbb{A}^{*}} \mathbb{A}^{*} N .
$$


Let $\mathscr{Y}$ be the stable homotopy category, and let $\mathscr{K}$ be the $K(n)$-local stable homotopy category. For a $K(n)$-local spectrum $X \in \mathscr{K}$, we define $\Lambda^{\prime \prime}(X)$ to be the full subcategory of the comma category $(\mathscr{\downarrow} \downarrow X)$, whose objects are maps $X^{\prime \prime} \rightarrow X$ from finite spectra $X^{\prime \prime}$ of type at least $n$. Then $\Lambda^{\prime \prime}(X)$ is an essentially small filtered category (see [Hovey and Strickland 1999, §9] and [Hovey et al. 1997, §2.3]). For a spectrum $W \in \mathscr{Y}$, we set $\Lambda(W)=\Lambda^{\prime \prime}\left(L_{K(n)} W\right)$. The following lemma gives a sufficient condition that we can describe a generalized cohomology group of $W$ in terms of cohomology groups of $W_{\lambda}$ for $\lambda \in \Lambda(W)$.

Lemma 3.13. Let $R$ be a $K(n)$-local commutative ring spectrum. Suppose that the coefficient ring $R^{*}$ is even-periodic and $R^{0}$ is a linearly compact Noetherian ring. Then there is a natural isomorphism

$$
R^{*}(W) \cong \lim _{\lambda} R^{*}\left(W_{\lambda}\right)
$$

for any $W \in \mathscr{Y}$, where the inverse limit is taken over $\lambda \in \Lambda(W)$.

Proof. For $W \in \mathscr{S}$, we set $F^{*}(W)=\lim _{\lambda} R^{*}\left(W_{\lambda}\right)$. Note that $R^{*}(W) \cong R^{*}\left(L_{K(n)} W\right)$ for any $W \in \mathscr{S}$ since $R$ is $K(n)$-local. Then it is sufficient to show that $R^{*}(X) \cong$ $F^{*}(X)$ for any $X \in \mathscr{K}$. By the assumption of the coefficient ring $R^{*}$, the functor $R^{*}(-)$ on the category of finite spectra takes values in the category of linearly compact $R^{*}$-modules and continuous maps. Then $F^{*}(-)$ is a cohomology theory on $\mathscr{Y}$ by [Hovey et al. 1997, Proposition 2.3.16] and [Hovey and Strickland 1999, Proposition 9.2]. There is a natural transformation $R^{*}(-) \rightarrow F^{*}(-)$ of cohomology theories, which induces an isomorphism

$$
R^{*}\left(X^{\prime \prime}\right) \stackrel{\cong}{\rightrightarrows} F^{*}\left(X^{\prime \prime}\right)
$$

for any finite spectrum $X^{\prime \prime}$ of type at least $n$. Since $L_{K(n)} F(n)$ is a graded weak generator of $\mathscr{K}$ for any finite spectrum $F(n)$ of type $n$ ([Hovey and Strickland 1999, Theorem 7.3]), we obtain that $R^{*}(X) \stackrel{\cong}{\rightarrow} F^{*}(X)$ for any $X \in \mathcal{K}$.

Definition 3.14. For a finite spectrum $X$ of type at least $n, E_{n+1}^{*}(X)$ is annihilated by a power of $I_{n}$, and $\mathbb{A}^{*}(X) \cong E_{n+1}^{*}(X)\left[u_{n}^{-1}\right]$ is a module over $\mathbb{A}^{*} / J_{n}^{r}=$ $E_{n+1}^{*} / I_{n}^{r}\left[u_{n}^{-1}\right]$ for some $r$. We give a topology on $\mathbb{A}^{*}(X)$ as in Definition 3.6. For a spectrum $W, \mathbb{A}^{*}(W) \cong \lim _{\varkappa} \mathbb{A}^{*}\left(W_{\lambda}\right)$ by Lemma 3.13 , where $W_{\lambda}$ are finite spectra of type at least $n$. We give a topology on $\mathbb{A}^{*}(W)$ by the inverse limit topology.

For a compact space $T$ and a finite spectrum $X$ of type at least $n$,

$$
\operatorname{Map}_{c}\left(T, \mathbb{A}^{*}(X)\right) \cong \operatorname{Map}_{c}\left(T, \mathbb{A}^{*}\right) \otimes_{\mathbb{A}^{*}} \mathbb{A}^{*}(X)
$$

by Proposition 3.10, and $\operatorname{Map}_{c}\left(T, \mathbb{A}^{*}\right)$ is profree by Proposition 3.8. To study the functor $\operatorname{Map}_{c}\left(T, \mathbb{A}^{*}(-)\right)$ on the stable homotopy category $\mathscr{Y}$, we consider the 
following functor. Let $F$ be a profree $\mathbb{A}^{*}$-module. We define a functor $H_{F}(-)$ from the stable homotopy category $\mathscr{S}$ to the category of $\mathbb{A}^{*}$-modules by

$$
H_{F}(W)=\lim _{\lambda} F \otimes_{\mathbb{A}^{*}} \mathbb{A}^{*}\left(W_{\lambda}\right),
$$

where the inverse limit is taken over $\lambda \in \Lambda(W)$.

Lemma 3.15. The functor $H_{F}(-)$ is a cohomology theory on $\mathscr{Y}$.

Proof. Since $F$ is a direct summand of some product $\prod_{\alpha} A^{*}$ by [Hovey and Strickland 1999, Proposition A.13], it is sufficient to show that the functor $Z \mapsto$ $\lim _{\lambda}\left(\prod_{\alpha} \mathbb{A}^{*}\right) \otimes_{\mathbb{A}^{*}} \mathbb{A}^{*}\left(W_{\lambda}\right)$ is a cohomology theory. Since $\mathbb{A}^{*}\left(W_{\lambda}\right)$ is finitely presented, $\left(\prod_{\alpha} \mathbb{A}^{*}\right) \otimes_{\mathbb{A}^{*}} \mathbb{A}^{*}\left(W_{\lambda}\right) \cong \prod_{\alpha} \mathbb{A}^{*}\left(W_{\lambda}\right)$ by Lemma 3.11. Hence

$$
\lim _{\lambda}\left(\prod_{\alpha} \mathbb{A}^{*}\right) \otimes_{\mathbb{A}^{*}} \mathbb{A}^{*}\left(W_{\lambda}\right) \cong \prod_{\alpha} \mathbb{A}^{*}(W),
$$

and $\prod_{\alpha} A^{*}(W)$ is a cohomology theory. This completes the proof.

The following theorem will be used to identify the $E_{2}$-term of the $K(n)$-localization of the $K(n+1)$-local $E_{n+1}$-Adams spectral sequence to the continuous cohomology group of $G_{n+1}$ in Section 4 below.

Theorem 3.16. For any compact space $T$, the functor $\operatorname{Map}_{c}\left(T, \mathbb{A}^{*}(-)\right)$ is a cohomology theory.

Proof. By Proposition 3.10, there is a natural isomorphism

$$
\operatorname{Map}_{c}\left(T, \mathbb{A}^{*}(W)\right) \cong \lim _{\lambda} \operatorname{Map}_{c}\left(T, \mathbb{A}^{*}\right){\underset{A^{*}}{\otimes}}_{\mathbb{A}^{*}}\left(W_{\lambda}\right) .
$$

But $\operatorname{Map}_{c}\left(T, A^{*}\right)$ is profree by Proposition 3.8. Therefore the theorem follows from Lemma 3.15.

\section{Construction of the spectral sequence}

We set $\widehat{S}=L_{K(n)} L_{K(n+1)} S^{0}$. In this section we construct a spectral sequence which converges strongly and conditionally to $[W, \widehat{S}]^{*}$ for any spectrum $W$ by applying the $K(n)$-localization functor to the $K(n+1)$-local $E_{n+1}$-Adams resolution of $L_{K(n+1)} S^{0}$. Then we describe the $E_{2}$-term in terms of the continuous cohomology group of $G_{n+1}$ with coefficients in $\mathbb{A}^{*}(W)$.

Let $E_{n}^{\wedge s}$ be the $K(n)$-localization of the smash product of $s$-copies of $E_{n}$

$$
E_{n}^{\wedge s}=L_{K(n)}(\overbrace{E_{n} \wedge \cdots \wedge E_{n}}^{s}) .
$$

The commutative ring spectrum structure on $E_{n}$ gives $E_{n}^{\wedge \bullet+1}=\left\{E_{n}^{\wedge s+1}\right\}_{s \geq 0}$ a cosimplicial $K(n)$-local commutative ring spectrum structure with augmentation 
$L_{K(n)} S^{0} \stackrel{\varepsilon}{\rightarrow} E_{n}^{\wedge \bullet+1}$. Then the associated cochain complex

$$
* \rightarrow L_{K(n)} S^{0} \stackrel{\varepsilon}{\longrightarrow} E_{n} \stackrel{d}{\longrightarrow} E_{n}^{\wedge 2} \stackrel{d}{\longrightarrow} E_{n}^{\wedge 3} \stackrel{d}{\longrightarrow} \cdots
$$

is a $K(n)$-local $E_{n}$-Adams resolution of $L_{K(n)} S^{0}$ in the sense of [Miller 1981; Devinatz and Hopkins 2004]. We denote the sequence (4-1) by $\operatorname{Res}\left(E_{n} ; L_{K(n)} S^{0}\right)$. There is an associated diagram of exact triangles

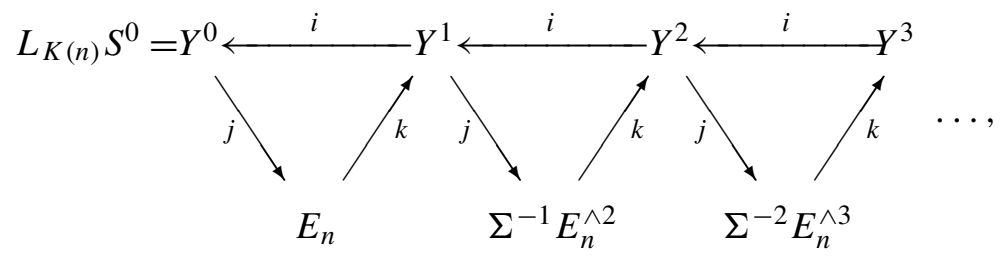

in the $K(n)$-local stable homotopy category, where $k$ has degree -1 and $j k=d$. We denote by $\operatorname{Ad}\left(E_{n} ; L_{K(n)} S^{0}\right)$ the diagram of exact triangles (4-2).

For any spectrum $W$, by applying the functor $[W,-]^{*}$ to $\operatorname{Ad}\left(E_{n} ; L_{K(n)} S^{0}\right)$ we obtain a $K(n)$-local $E_{n}$-Adams spectral sequence

$$
L_{K(n)} E_{r}^{s, t}(W) \Longrightarrow\left[W, L_{K(n)} S^{0}\right]^{s+t}
$$

with $L_{K(n)} E_{2}^{s, t}(W) \cong H_{c}^{s}\left(G_{n} ; E_{n}^{t}(W)\right)$. This spectral sequence converges strongly and conditionally. Furthermore, since $L_{K(n)} S^{0}$ is $K(n)$-local $E_{n}$-nilpotent [Devinatz and Hopkins 2004, Proposition A.3], the filtration (4-2) has the following property: There exists $N>0$ such that $Y^{s+N} \rightarrow Y^{s}$ is null for all $s \geq 0$. This property implies that there exist positive integers $r(n)$ and $s(n)$, which do not depend on $W$, such that $L_{K(n)} E_{r(n)}^{s, *}(W)=0$ for $s>s(n)$.

By applying the $K(n)$-localization functor to $\operatorname{Ad}\left(E_{n+1} ; L_{K(n+1)} S^{0}\right)$, we obtain the following diagram $L_{K(n)} \operatorname{Ad}\left(E_{n+1}, L_{K(n+1)} S^{0}\right)$ of exact triangles
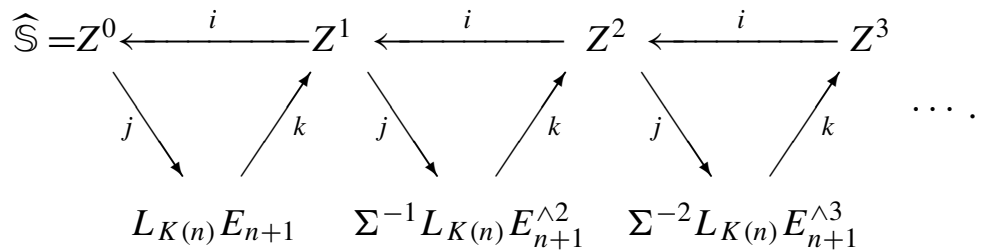

$$
L_{K(n)} E_{n+1} \quad \Sigma^{-1} L_{K(n)} E_{n+1}^{\wedge 2} \quad \Sigma^{-2} L_{K(n)} E_{n+1}^{\wedge 3}
$$

For any spectrum $W$, applying the functor $[W,-]^{*}$ to $L_{K(n)} \operatorname{Ad}\left(E_{n+1}, L_{K(n+1)} S^{0}\right)$, we obtain a spectral sequence

$$
L_{K(n)} L_{K(n+1)} E_{r}^{s, t}(W) \Longrightarrow[W, \widehat{\mathbb{S}}]^{s+t} .
$$

We call this spectral sequence the $K(n)$-localization of the $K(n+1)$-local $E_{n+1^{-}}$ Adams spectral sequence. 
Lemma 4.1. The spectral sequence $L_{K(n)} L_{K(n+1)} E_{r}^{s, t}(W) \Longrightarrow[W, \widehat{S}]^{s+t}$ converges conditionally and strongly for any spectrum $W$.

Proof. There exists $N>0$ such that $Y^{s+N} \rightarrow Y^{s}$ is null for all $s \geq 0$. Applying the $K(n)$-localization functor, we see that $Z^{s+N} \rightarrow Z^{s}$ is also null for all $s \geq 0$. This implies that the filtration of $[W, \widehat{S}]^{*}$ is finite. Hence the spectral sequence converges strongly by [Boardman 1999, Definition 5.2]. Also, we obtain that $\lim _{n}\left[W, Z^{n}\right]^{*}=\lim _{\longleftarrow}{ }^{1}\left[W, Z^{n}\right]^{*}=0$. Hence the spectral sequence converges conditionally by [Boardman 1999, Definition 5.10].

Remark 4.2. Note that there exist positive integers $r_{0}$ and $s_{0}$, which do not depend on $W$, such that $L_{K(n)} L_{K(n+1)} E_{r_{0}}^{s, *}(W)=0$ for $s>s_{0}$.

In the rest of this section we identify the $E_{2}$-term of the $K(n)$-localization of the $K(n+1)$-local $E_{n+1}$-Adams spectral sequence $L_{K(n)} L_{K(n+1)} E_{r}^{s, t}(W)$ with the continuous cohomology group of $G_{n+1}$ with coefficients in $\mathbb{A}^{*}(W)$. Let $C(s)=$ $E_{n+1}^{\wedge s+1}$. The $E_{1}$-term of the spectral sequence is given by $E_{1}^{s, t}=\left[W, L_{K(n)} C(s)\right]^{t}$. There is an isomorphism $C(s)^{*} \cong \operatorname{Map}_{c}\left(G_{n+1}^{s}, E_{n+1}^{*}\right)$ (see [Devinatz and Hopkins $2004, \S 2])$. Then we see that $C(s)^{*}$ is profree over $E_{n+1}^{*}$ by Proposition 3.3. The following lemma gives a similar description for $L_{K(n)} C(s)^{*}$.

Lemma 4.3. For $s \geq 0$, we have $L_{K(n)} C(s)^{*} \cong \operatorname{Map}_{c}\left(G_{n+1}^{s}, A^{*}\right)$.

Proof. There is a tower $\{M(J)\}_{J}$ of generalized Moore spectra of type $n$ as in [Hovey and Strickland 1999, Proposition 4.2] such that $L_{K(n)} W \simeq \operatorname{holim}_{J} L_{n} W \wedge$ $M(J)$ for any spectrum $W$ [Hovey and Strickland 1999, Proposition 7.10(e)]. Since $C(s)$ is Landweber exact of height $(n+1)$, we obtain that $L_{K(n)} C(s)^{*} \cong \mathbb{A}^{*} C(s)^{*}$. Then $\mathbb{A}^{*} C(s)^{*} \cong \operatorname{Map}_{c}\left(G_{n+1}^{s}, \mathbb{A}^{*}\right)$ by Lemma 3.7, since

$$
C(s)^{*} \cong \operatorname{Map}_{c}\left(G_{n+1}^{s}, E_{n+1}^{*}\right) .
$$

Corollary 4.4. For $s \geq 0, L_{K(n)} C(s)^{*}$ is Landweber exact and profree over $\mathbb{A}^{*}$.

Proof. This follows from Proposition 3.8 and Corollary 3.9.

Then we obtain a description for the $E_{1}$-term $\left[W, L_{K(n)} C(s)\right]^{*}$ as a module of continuous maps from $G_{n+1}^{s}$ to $\mathbb{A}^{*}(W)$.

Proposition 4.5. For any spectrum $W$, there is a natural isomorphism

$$
\left[W, L_{K(n)} C(s)\right]^{*} \cong \operatorname{Map}_{c}\left(G_{n+1}^{s}, \mathbb{A}^{*}(W)\right) .
$$

Proof. By Lemma 4.3 and Corollary $4.4, L_{K(n)} C(s)^{*} \cong \operatorname{Map}_{c}\left(G_{n+1}^{s}, A^{*}\right)$ is Landweber exact. Then there is a natural isomorphism

$$
\left[W, L_{K(n)} C(s)\right]^{*} \cong \operatorname{Map}_{c}\left(G_{n+1}^{s}, \mathbb{A}^{*}\right) \otimes_{\mathbb{A}^{*}} \mathbb{A}^{*}(W)
$$

for any finite spectrum $W$. By Proposition 3.10, the right hand side is isomorphic to $\operatorname{Map}_{c}\left(G_{n+1}^{s}, \mathbb{A}^{*}(W)\right)$. Since $\operatorname{Map}_{c}\left(G_{n+1}^{s}, \mathbb{A}^{*}\right)$ is even concentrated, there is a 
unique extension to a cohomology theory for any spectra by [Hovey and Strickland 1999, Theorem 2.8]. Obviously, $\left[-, L_{K(n)} C(s)\right]^{*}$ is such an extension. On the other hand, $\operatorname{Map}_{c}\left(G_{n+1}^{s}, \mathbb{A}^{*}(-)\right)$ is also an extension by Theorem 3.16. Therefore $\left[W, L_{K(n)} C(s)\right]^{*} \cong \operatorname{Map}_{c}\left(G_{n+1}^{s}, \mathbb{A}^{*}(W)\right)$ for any spectrum $W$.

For a topological group $G$ and a topological $G$-module $M$, denote by $C_{c}^{*}(G ; M)$ the continuous cochain complex of $G$ with coefficients in $M$. Define $H_{c}^{*}(G ; M)$ to be the cohomology group of $C_{c}^{*}(G ; M)$, and call it the continuous cohomology of $G$ with coefficients in $M$. Let $[W, C(*)]^{t}$ be the cochain complex associated with the cosimplicial abelian group $[W, C(\bullet)]^{t}$. Then there is a natural isomorphism $[W, C(*)]^{t} \cong C_{c}^{*}\left(G_{n+1}, E_{n+1}^{t}(W)\right)$ of cochain complexes [Devinatz and Hopkins 2004, §4]. By Proposition 4.5, this implies a natural isomorphism $\left[W, L_{K(n)} C(*)\right]^{t} \cong C_{c}^{*}\left(G_{n+1}, \mathbb{A}^{t}(W)\right)$ of cochain complexes. Hence we obtain the following corollary.

Corollary 4.6. For any spectrum $W$, there is a natural isomorphism

$$
H^{s}\left(\left[W, L_{K(n)} C(*)\right]^{t}\right) \cong H_{c}^{s}\left(G_{n+1} ; \mathbb{A}^{t}(W)\right) .
$$

As a summary we obtain the following theorem.

Theorem 4.7. For any spectrum $W$, there is a natural spectral sequence

$$
L_{K(n)} L_{K(n+1)} E_{r}^{s, t}(W)
$$

which converges strongly and conditionally to $[W, \widehat{S}]^{*}$ :

$$
L_{K(n)} L_{K(n+1)} E_{2}^{s, t}(W) \Longrightarrow[W, \widehat{\mathbb{S}}]^{s+t} .
$$

The $E_{2}$-term is given by

$$
L_{K(n)} L_{K(n+1)} E_{2}^{s, t}(W) \cong H_{c}^{s}\left(G_{n+1} ; \mathbb{A}^{t}(W)\right) .
$$

Furthermore, there exist positive integers $r_{0}$ and $s_{0}$ such that

$$
L_{K(n)} L_{K(n+1)} E_{r_{0}}^{s, *}(W)=0
$$

for $s>s_{0}$, where $r_{0}$ and $s_{0}$ do not depend on $W$.

\section{The cohomology group $H_{c}^{*}\left(\mathbb{G} ; \mathbb{B}^{*}(W)\right)$}

In this section we introduce a cohomology group $\boldsymbol{H}_{c}^{*}\left(\mathbb{G} ; \mathbb{B}^{*}(W)\right)$ of $\mathbb{G}$ with coefficients in $\mathbb{B}^{*}(W)$ for a spectrum $W$. Then we show that $\boldsymbol{H}_{c}^{*}\left(\mathbb{G} ; \mathbb{B}^{*}(W)\right)$ is naturally isomorphic to the continuous cohomology group $H_{c}^{*}\left(G_{n+1} ; \mathbb{A}^{*}(W)\right)$ of $G_{n+1}$ with coefficients in $\mathbb{A}^{*}(W)$. The cohomology group $\boldsymbol{H}_{c}^{*}\left(\mathbb{G} ; \mathbb{B}^{*}(W)\right)$ will be used to connect the $E_{2}$-term of the $K(n)$-local $E_{n}$-Adams spectral sequence for $W$ and 
the $E_{2}$-term of the $K(n)$-localization of the $K(n+1)$-local $E_{n+1}$-Adams spectral sequence for $W$ in Section 7 below.

First we introduce a topology for modules of continuous maps from a profinite group to an $\mathbb{A}^{*}$-module of certain type. Then we study a continuous cohomology group of a profinite group with coefficients in such a topological module of mappings.

Definition 5.1. Let $G$ be a profinite group. Suppose that $M=\lim _{\lambda} \mathbb{A}^{*} N_{\lambda}$ with the inverse limit topology, where $\left\{N_{\lambda}\right\}_{\lambda \in \Lambda}$ is a cofiltered system of finitely generated $E_{n+1}^{*}$-modules. By Lemma 3.7, there is an isomorphism

$$
\operatorname{Map}_{c}(G, M) \cong \lim _{\lambda} \mathbb{A}^{*} \operatorname{Map}_{c}\left(G, N_{\lambda}\right) .
$$

We give a topology on $\mathbb{A}^{*} \operatorname{Map}_{c}\left(G, N_{\lambda}\right)$ as in Definition 3.6. Then we give a topology on $\operatorname{Map}_{c}(G, M)$ by the inverse limit topology. For any spectrum $W$, $\mathbb{A}^{*}(W) \cong \lim _{\lambda} \mathbb{A}^{*} E_{n+1}^{*}\left(W_{\lambda}\right)$ by Lemma 3.13 , where $W_{\lambda}$ are finite spectra of type at least $n$. We give a topology on $\operatorname{Map}_{c}\left(G, A^{*}(W)\right)$ as above.

The following lemma shows that the mapping spaces have an expected adjunction property.

Lemma 5.2. Let $G$ and $H$ be profinite groups. Suppose that $M=\lim _{\varkappa_{\lambda}} A^{*} N_{\lambda}$ with the inverse limit topology, where $\left\{N_{\lambda}\right\}_{\lambda \in \Lambda}$ is a cofiltered system of finitely generated $E_{n+1}^{*}$-modules. Then there is an isomorphism

$$
\operatorname{Map}_{c}\left(G, \operatorname{Map}_{c}(H, M)\right) \cong \operatorname{Map}_{c}(G \times H, M) .
$$

Proof. We have

$$
\begin{gathered}
\operatorname{Map}_{c}\left(G, \operatorname{Map}_{c}(H, M)=\lim _{\lambda} \operatorname{Map}_{c}\left(G, \operatorname{Map}_{c}\left(H, \mathbb{A}^{*} N_{\lambda}\right),\right.\right. \\
\operatorname{Map}_{c}(G \times H, M)=\lim _{\lambda} \operatorname{Map}_{c}\left(G \times H, \mathbb{A}^{*} N_{\lambda}\right) .
\end{gathered}
$$

Hence it is sufficient to show that the lemma holds when $M=\mathbb{A}^{*} N$ with finitely generated $N$. Suppose that $N$ is a finitely generated $E_{n+1}^{*}$-module. Let $N_{r}$ be the image of the localization map $N / I_{n}^{r} N \rightarrow N / I_{n}^{r} N\left[u_{n}^{-1}\right]$, and let $L_{r}=\operatorname{Map}_{c}\left(H, N_{r}\right)$. Note that $N_{r}$ and $L_{r}$ are $\left(u_{n}\right)$-torsion free. By Lemma 3.5, $\operatorname{Map}_{c}\left(H, \mathbb{A}^{*} N\right)=$ $\lim _{r} L_{r}\left[u_{n}^{-1}\right]$. Then $\operatorname{Map}_{c}\left(G, \operatorname{Map}_{c}\left(H, \mathbb{A}^{*} N\right)\right)=\lim _{r} \operatorname{Map}_{c}\left(G, L_{r}\left[u_{n}^{-1}\right]\right)$. Again by Lemma 3.5, we have $\operatorname{Map}_{c}\left(G, L_{r}\left[u_{n}^{-1}\right]\right)=\operatorname{Map}_{c}\left(G, L_{r}\right)\left[u_{n}^{-1}\right]$. The fact that $N_{r}$ is a profinite module implies that $\operatorname{Map}_{c}\left(G, L_{r}\right)=\operatorname{Map}_{c}\left(G \times H, N_{r}\right)$. By Lemma 3.5, we obtain $\lim _{r} \operatorname{Map}_{c}\left(G \times H, N_{r}\right)\left[u_{n}^{-1}\right]=\operatorname{Map}_{c}\left(G \times H, \mathbb{A}^{*} N\right)$.

Corollary 5.3. Let $G$ and $H$ be profinite groups. For any spectrum $W$, there is a natural isomorphism

$$
\left.\operatorname{Map}_{c}\left(G, \operatorname{Map}_{c}\left(H, \mathbb{A}^{*}(W)\right)\right)\right) \cong \operatorname{Map}_{c}\left(G \times H, \mathbb{A}^{*}(W)\right) .
$$


Suppose that a profinite group $G$ continuously acts on a topological module $M$ from the right. For $q>0$, we define a right $G$-action on $\operatorname{Map}_{c}(G, M)$ by

$$
\varphi^{g}\left(h_{1}, \ldots, h_{q}\right)=\varphi\left(h_{1} g^{-1}, \ldots, h_{q} g^{-1}\right)^{g},
$$

where $\varphi \in \operatorname{Map}_{c}\left(G^{q}, M\right)$ and $g, h_{1}, \ldots, h_{q} \in G$. Then $\operatorname{Map}_{c}\left(G^{q}, M\right)$ is a topological $G$-module. The following proposition shows that the coinduced module $\operatorname{Map}_{c}\left(G^{q}, M\right)$ is acyclic with respect to $H_{c}^{*}(G ;-)$.

Proposition 5.4. Let $G$ be a profinite group. Suppose that $M=\lim _{\lambda} \mathbb{A}^{*} N_{\lambda}$ with the inverse limit topology, where $\left\{N_{\lambda}\right\}_{\lambda \in \Lambda}$ is a cofiltered system of finitely generated $E_{n+1}^{*}$-modules. Furthermore, suppose that $G$ continuously acts on $M$. For $p>0$ and $q>0$, we have $H_{c}^{p}\left(G ; \operatorname{Map}_{c}\left(G^{q}, M\right)\right)=0$, and $H_{c}^{0}\left(G ; \operatorname{Map}_{c}\left(G^{q}, M\right)\right)=$ $\operatorname{Map}_{c}\left(G^{q}, M\right)^{G}$.

Proof. Set

$$
C_{c}^{-1}\left(G ; \operatorname{Map}_{c}\left(G^{q}, M\right)\right)=\operatorname{Map}_{c}\left(G^{q}, M\right)^{G}, \quad C^{p, q}=C_{c}^{p}\left(G ; \operatorname{Map}_{c}\left(G^{q}, M\right)\right) .
$$

Then $C^{p, q} \cong \operatorname{Map}_{c}\left(G^{q} \times G^{p+1}, M\right)^{G}$ by Lemma 5.2. The boundary map $d^{p}$ : $C^{p, q} \rightarrow C^{p+1, q}$ is given by

$d^{p} f\left(h_{1}, \ldots, h_{q} ; g_{0}, \ldots, g_{p+1}\right)$

$$
=\sum_{i=0}^{p+1}(-1)^{i} f\left(h_{1}, \ldots, h_{q} ; g_{0}, \ldots, g_{i-1}, g_{i+1}, \ldots, g_{p+1}\right) .
$$

We define $s^{p}: C^{p, q} \rightarrow C^{p-1, q}$ by

$$
s^{p} f\left(h_{1}, \ldots, h_{q} ; g_{0}, \ldots, g_{p-1}\right)=f\left(h_{1}, \ldots, h_{q} ; h_{q}, g_{0}, \ldots, g_{p-1}\right) .
$$

Then we can verify that $s^{p+1} d^{p}(f)+d^{p-1} s^{p}(f)=f$ for any $f \in C^{p, q}$.

Corollary 5.5. Let $p>0$ and $q>0$. Then $H_{c}^{p}\left(G_{n+1} ; \operatorname{Map}_{c}\left(G_{n+1}^{q}, \mathbb{A}^{*}(W)\right)\right)=0$ and $H_{c}^{0}\left(G_{n+1} ; \operatorname{Map}_{c}\left(G_{n+1}^{q}, \mathbb{A}^{*}(W)\right)\right)=\operatorname{Map}_{c}\left(G_{n+1}^{q}, \mathbb{A}^{*}(W)\right)^{G_{n+1}}$ for any spectrum $W$.

Next we define a cohomology group $\boldsymbol{H}_{c}^{*}\left(\mathbb{G} ; \mathbb{B}^{*}(W)\right)$. For this purpose, we introduce a topology on $\mathbb{B}(i)^{*}(W)$.

Definition 5.6. For a spectrum $W, \mathbb{B}(i)^{*}(W)$ is a product of finite many copies of $\mathbb{A}^{*}(W)$ since $\mathbb{B}(i)^{*}$ is finitely generated free over $\mathbb{A}^{*}$. We give a topology on $\mathbb{B}(i)^{*}(W)$ by the product topology.

Recall that the group $\mathbb{G}=G_{n+1} \times_{\Gamma} G_{n}$ acts on the cohomology theory $\mathbb{B}^{*}(-)$ as multiplicative cohomology operations by Proposition 2.3. For $i \geq-1$, we set $\mathbb{G}(i)=G_{n+1} \times_{\Gamma} G_{n}(i)$, where $G_{n}(i)=\Gamma \ltimes S_{n}(i)$. Then $\mathbb{G}(i)$ acts on $\mathbb{B}(i)^{*}(W)$ naturally and continuously. Note that we can write $\mathbb{B}(i)^{*}(W)=\lim _{\lambda} \mathbb{A}^{*} N_{\lambda}$ with 
finitely generated $E_{n+1}^{*}$-modules $N_{\lambda}$ since $\mathbb{B}(i)^{*}$ is finitely generated free over $\mathbb{A}^{*}$. Then $\operatorname{Map}_{c}\left(\mathbb{G}(i)^{p+1}, \mathbb{B}(i)^{*}(W)\right)$ is a topological module for any $p \geq 0$ as in Definition 5.1.

Definition 5.7. For a spectrum $W$, we define a cochain complex $\boldsymbol{C}_{c}^{*}\left(\mathbb{G} ; \mathbb{B}^{*}(W)\right)$ by

$$
\boldsymbol{C}_{c}^{*}\left(\mathbb{G} ; \mathbb{B}^{*}(W)\right)=\lim _{\lambda} \lim _{i} C_{c}^{*}\left(\mathbb{G}(i) ; \mathbb{B}(i)^{*}\left(W_{\lambda}\right)\right),
$$

where the inverse limit is taken over $\lambda \in \Lambda(W)$. Then we define a cohomology group $\boldsymbol{H}_{c}^{*}\left(\mathbb{G} ; \mathbb{B}^{*}(W)\right)$ of $\mathbb{G}$ with coefficients in $\mathbb{B}^{*}(W)$ to be the cohomology group of $\boldsymbol{C}_{c}^{*}\left(\mathbb{G} ; \mathbb{B}^{*}(W)\right)$

$$
\boldsymbol{H}_{c}^{*}\left(\mathbb{G} ; \mathbb{B}^{*}(W)\right)=H^{*}\left(\boldsymbol{C}_{c}^{*}\left(\mathbb{G} ; \mathbb{B}^{*}(W)\right)\right) .
$$

Note that both of $\boldsymbol{C}_{c}^{*}\left(\mathbb{G} ; \mathbb{B}^{*}(W)\right)$ and $\boldsymbol{H}_{c}^{*}\left(\mathbb{G} ; \mathbb{B}^{*}(W)\right)$ are not functors of $\mathbb{B}^{*}(W)$ in spite of their notation.

For a continuous cochain complex $C_{c}^{*}\left(G_{n+1} ; \mathbb{A}^{*}(W)\right)$ of $G_{n+1}$ with coefficients in $A^{*}(W)$, there is an isomorphism

$$
C_{c}^{*}\left(G_{n+1} ; \mathbb{A}^{*}(W)\right) \cong \lim _{\lambda} C_{c}^{*}\left(G_{n+1} ; \mathbb{A}^{*}\left(W_{\lambda}\right)\right) .
$$

The canonical maps $\mathbb{A}^{*}\left(W_{\lambda}\right) \rightarrow \mathbb{B}(i)^{*}\left(W_{\lambda}\right)$ and the projections $\mathbb{G}(i) \rightarrow G_{n+1}$ define a cochain map

$$
C_{c}^{*}\left(G_{n+1} ; \mathbb{A}^{*}(W)\right) \longrightarrow C_{c}^{*}\left(\mathbb{G} ; \mathbb{B}^{*}(W)\right) .
$$

We call the induced map on cohomology groups an inflation map

$$
H_{c}^{*}\left(G_{n+1} ; \mathbb{A}^{*}(W)\right) \longrightarrow \boldsymbol{H}_{c}^{*}\left(\mathbb{G} ; \mathbb{B}^{*}(W)\right) .
$$

In the rest of this section we prove the following theorem.

Theorem 5.8. The inflation map $H_{c}^{*}\left(G_{n+1} ; \mathbb{A}^{*}(W)\right) \rightarrow \boldsymbol{H}_{c}^{*}\left(\mathbb{G} ; \mathbb{B}^{*}(W)\right)$ is an isomorphism for any spectrum $W$.

By definition, $\boldsymbol{H}_{c}^{*}\left(\mathbb{G} ; \mathbb{B}^{*}(W)\right)$ is the cohomology group of the inverse limit of the cochain complexes $\lim _{i} C_{c}^{*}\left(\mathbb{G}(i) ; \mathbb{B}(i)^{*}\left(W_{\lambda}\right)\right)$. For the cohomology group of the inverse limit of cochain complexes $\left\{C_{\lambda}^{*}\right\}_{\lambda \in \Lambda}$, we have a spectral sequence to describe it in terms of the cohomology groups of $C_{\lambda}^{*}$ under suitable circumstances.

Lemma 5.9. Let $\left\{C_{\lambda}^{*}\right\}_{\lambda \in \Lambda}$ be a system of cochain complexes indexed by a small category $\Lambda$. We assume that $\lim _{\lambda}^{j} C_{\lambda}^{*}=0$ for $j>0$. Then there is a spectral sequence

$$
E_{2}^{s, t}=\lim _{\lambda}^{s} H^{t}\left(C_{\lambda}^{*}\right) \Longrightarrow H^{s+t}\left(\lim _{\lambda} C_{\lambda}^{*}\right)
$$


Proof. Let $\prod^{*} C_{\lambda}^{*}$ be the double complex associated to the cosimplicial replacement [Bousfield and Kan 1972, XI.5] of $\left\{C_{\lambda}^{*}\right\}$. Then we have two spectral sequences

$$
\begin{aligned}
& {\underset{\lim }{\longleftarrow}}_{\lambda}^{s} H^{t}\left(C_{\lambda}^{*}\right) \Longrightarrow H^{s+t}\left(\prod^{*} C_{\lambda}^{*}\right), \\
& H^{s}\left(\lim _{\lambda}^{t} C_{\lambda}^{*}\right) \Longrightarrow H^{s+t}\left(\prod^{*} C_{\lambda}^{*}\right) .
\end{aligned}
$$

By the assumption, the second spectral sequence collapses to give $H^{*}\left(\lim _{\lambda} C_{\lambda}^{*}\right) \cong$ $H^{*}\left(\prod^{*} C_{\lambda}^{*}\right)$. Hence the first spectral sequence gives the desired one.

The next lemma gives a sufficient condition for all the higher inverse limits to vanish.

Lemma 5.10. Let $F$ be a profree $\mathbb{A}^{*}$-module. Then $\lim _{\lambda}^{j} F \otimes_{\mathbb{A}^{*}} \mathbb{A}^{*}\left(W_{\lambda}\right)=0$ for $j>0$.

Proof. Since $F$ is a direct summand of some product of (suspensions of) $\mathbb{A}^{*}$ by [Hovey and Strickland 1999, Proposition A.13], we may assume that $F=\prod_{\alpha} A^{*}$. For a finite spectrum $W_{\lambda}, F \otimes \mathbb{A}^{*}\left(W_{\lambda}\right) \cong \prod_{\alpha} \mathbb{A}^{*}\left(W_{\lambda}\right)$ since $\mathbb{A}^{*}\left(W_{\lambda}\right)$ is a finitely presented $\mathbb{A}^{*}$-module. Then we have $\lim _{\varkappa_{\lambda}^{j}}^{j} \prod_{\alpha} \mathbb{A}^{*}\left(W_{\lambda}\right) \cong \prod_{\alpha} \lim _{\lambda}^{j} \mathbb{A}^{*}\left(W_{\lambda}\right)$. The lemma follows from the fact that $\lim _{\lambda}^{j} \mathbb{A}^{*}\left(W_{\lambda}\right)=0$ for $j>0$ since $\mathbb{A}^{*}\left(W_{\lambda}\right)$ is a linearly compact $\mathbb{A}^{*}$-module for all $\lambda$.

By Proposition 3.8, $\operatorname{Map}_{c}\left(G_{n+1}^{q+1} ; \mathbb{A}^{*}\right)$ and $\operatorname{Map}_{c}\left(\mathbb{G}(i)^{q+1}, \mathbb{B}(i)^{*}\right)$ are profree $\mathbb{A}^{*}$ modules. Then the completion of $\lim _{i} C_{c}^{*}\left(\mathbb{G}(i) ; \mathbb{B}(i)^{*}\right)$ at $I_{n}$ is also a profree $\mathbb{A}^{*}$-module. By Lemma 5.10, we obtain that $\lim _{\lambda}^{j} C_{c}^{*}\left(G_{n+1} ; \mathbb{A}^{*}\left(W_{\lambda}\right)\right)=0$ and $\lim _{\lambda}^{j} \lim _{i} C_{c}^{*}\left(\mathbb{G}(i) ; \mathbb{B}(i)^{*}\left(W_{\lambda}\right)\right)=0$ for $j>0$. Hence, by Lemma 5.9, we obtain two spectral sequences

$$
\begin{aligned}
{ }_{I} E_{2}^{s, t} & =\lim _{\lambda}^{s} H_{c}^{t}\left(G_{n+1} ; \mathbb{A}^{*}\left(W_{\lambda}\right)\right) \quad \Longrightarrow H_{c}^{*}\left(G_{n+1} ; \mathbb{A}^{*}(W)\right), \\
{ }_{I I} E_{2}^{s, t} & =\stackrel{\lim }{\longleftarrow}_{\lambda}^{s} \underline{\lim }_{i} H_{c}^{t}\left(\mathbb{G}(i) ; \mathbb{B}(i)^{*}\left(W_{\lambda}\right)\right) \Longrightarrow \boldsymbol{H}_{c}^{*}\left(\mathbb{G} ; \mathbb{B}^{*}(W)\right) .
\end{aligned}
$$

The system of cochain maps

$$
\left\{C_{c}^{*}\left(G_{n+1} ; \mathbb{A}^{*}\left(W_{\lambda}\right)\right)\right\}_{\lambda} \longrightarrow\left\{\underline{\lim }_{i} C_{c}^{*}\left(\mathbb{G}(i) ; \mathbb{B}(i)^{*}\left(W_{\lambda}\right)\right)\right\}_{\lambda}
$$

induces a morphism of spectral sequences

$$
f_{r}:{ }_{I} E_{r}^{*, *} \longrightarrow{ }_{I I} E_{r}^{*, *}
$$

which converges to the inflation map (5-1).

We show that this morphism of spectral sequences is an isomorphism from the $E_{2}$-terms onward. For this purpose, it is sufficient to show that the inflation map $H_{c}^{*}\left(G_{n+1} ; \mathbb{A}^{*}(W)\right) \rightarrow H_{c}^{*}\left(\mathbb{G}(i) ; \mathbb{B}(i)^{*}(W)\right)$ is an isomorphism for $i \geq 0$. We shall construct two acyclic resolutions $I^{*}(W)$ and $J^{*}(i, W)$ of $\mathbb{A}^{*}(W)$ with respect to $H_{c}^{*}\left(G_{n+1} ;-\right)$ so that

$$
I^{*}(W)^{G_{n+1}} \cong C_{c}^{*}\left(G_{n+1} ; \mathbb{A}^{*}(W)\right) \quad \text { and } \quad J^{*}(i, W)^{G_{n+1}} \cong C_{c}^{*}\left(\mathbb{G}(i) ; \mathbb{B}(i)^{*}(W)\right) .
$$


We shall enlarge the complexes $C_{c}^{*}\left(G_{n+1} ; \mathbb{A}^{*}(W)\right)$ and $C_{c}^{*}\left(\mathbb{G}(i) ; \mathbb{B}(i)^{*}(W)\right)$ to double complexes $C_{c}^{*}\left(G_{n+1} ; I^{*}(W)\right)$ and $C_{c}^{*}\left(G_{n+1} ; J(i, W)\right)$. We shall construct a map of double complexes $C_{c}^{*}\left(G_{n+1} ; I^{*}(W)\right) \rightarrow C_{c}^{*}\left(G_{n+1} ; J(i, W)\right)$, which induces the inflation map $H_{c}^{*}\left(G_{n+1} ; \mathbb{A}^{*}(W)\right) \rightarrow H_{c}^{*}\left(\mathbb{G}(i) ; \mathbb{B}(i)^{*}(W)\right)$. Then we shall show that the map of double complexes induces an isomorphism on cohomology groups.

First, we construct an acyclic resolution $I^{*}(W)$ of $A^{*}(W)$. We set

$$
I^{q}(W)=\operatorname{Map}_{c}\left(G_{n+1}^{q+1}, A^{*}(W)\right)
$$

the topological $\mathbb{A}^{*}$-module of all continuous maps from $G_{n+1}^{q+1}$ to $\mathbb{A}^{*}(W)$. Define a $\operatorname{map} d^{q}: I^{q}(W) \rightarrow I^{q+1}(W)$ by

$$
d^{q}(f)\left(g_{0}, \ldots, g_{q+1}\right)=\sum_{j=0}^{q+1}(-1)^{j} f\left(g_{0}, \ldots, g_{j-1}, g_{j+1}, \ldots, g_{q+1}\right) .
$$

Then $I^{*}(W)=\left\{I^{q}(W), d^{q}\right\}_{q \geq-1}$ forms an augmented cochain complex satisfying $I^{-1}(W)=\mathbb{A}^{*}(W)$. The group $G_{n+1}$ acts on the cochain complex $I^{*}(W)$ and

$$
I^{*}(W)^{G_{n+1}} \cong C_{c}^{*}\left(G_{n+1} ; \mathbb{A}^{*}(W)\right) .
$$

Lemma 5.11. For $p>0$ and $q \geq 0$, we have

$$
H_{c}^{p}\left(G_{n+1} ; I^{q}(W)\right)=0 \quad \text { and } \quad H_{c}^{0}\left(G_{n+1} ; I^{q}(W)\right)=C_{c}^{q}\left(G_{n+1} ; \mathbb{A}^{*}(W)\right) .
$$

The sequence $0 \rightarrow \mathbb{A}^{*}(W) \stackrel{d^{-1}}{\rightarrow} I^{0}(W) \stackrel{d^{1}}{\rightarrow} I^{1}(W) \stackrel{d^{2}}{\rightarrow} \cdots$ is a split exact sequence of topological $\mathbb{A}^{*}$-modules. Hence $I^{*}(W)$ is an acyclic resolution of $\mathbb{A}^{*}(W)$ with respect to $H_{c}^{*}\left(G_{n+1} ;-\right)$.

Proof. Since $I^{q}(W)=\operatorname{Map}_{c}\left(G_{n+1}^{q+1}, \mathbb{A}^{*}(W)\right)$, the first assertion is a consequence of Corollary 5.5. We define $s^{q}: I^{q}(W) \rightarrow I^{q-1}(W)$ by $s^{q}(f)\left(g_{0}, \ldots, g_{q-1}\right)=$ $f\left(e, g_{0}, \ldots, g_{q-1}\right)$. Then we can verify that $\left\{s^{q}\right\}_{q \geq 0}$ gives a desired splitting.

Next we construct another acyclic resolution $J^{*}(i, W)$ of $\mathbb{A}^{*}(W)$. We set

$$
J^{q}(i, W)=\operatorname{Map}_{c}\left(\mathbb{G}(i)^{q+1}, \mathbb{B}(i)^{*}(W)\right)^{S_{n}(i)} .
$$

the topological $\mathbb{A}^{*}$-module of all $S_{n}(i)$-equivariant continuous maps from $\mathbb{G}(i)^{q+1}$ to $\mathbb{B}(i)^{*}(W)$. Define a map $d^{q}: J^{q}(i, W) \rightarrow J^{q+1}(i, W)$ by

$$
d^{q} f\left(g_{0}, \ldots, g_{p+1}\right)=\sum_{j=0}^{p+1}(-1)^{j} f\left(g_{0}, \ldots, g_{j-1}, g_{j}, \ldots, g_{p+1}\right) .
$$

Then $J^{*}(i, W)=\left\{J^{q}(i, W), d^{q}\right\}_{q \geq-1}$ forms an augmented cochain complex with $J^{-1}(i, W)=\mathbb{A}^{*}(W)$. The group $G_{n+1}$ acts on $J^{*}(i, W)$ and

$$
J^{*}(i, W)^{G_{n+1}} \cong C_{c}^{*}\left(\mathbb{G}(i) ; \mathbb{B}(i)^{*}(W)\right) .
$$


We compare $J^{*}(i, W)$ with $I^{*}(W)$. Let $D^{*}=C^{*}\left(S_{n}(i) ; \mathbb{B}(i)^{*}\right)$ be the cochain complex of $S_{n}(i)$ with coefficients in $\mathbb{B}(i)^{*}$. Since $\mathbb{A}^{*} \rightarrow \mathbb{B}(i)^{*}$ is a Galois extension with Galois group $S_{n}(i)$, there is an isomorphism $D^{q} \cong \mathbb{B}(i)^{* \otimes(q+1)}$. Then the differential $d^{q}: D^{q} \rightarrow D^{q+1}$ corresponds to $d^{q}: \mathbb{B}(i)^{*(q+1)} \rightarrow \mathbb{B}(i)^{*(q+2)}$ given by

$$
d^{q}\left(b_{0} \otimes \cdots \otimes b_{q}\right)=\sum_{j=0}^{q}(-1)^{j} b_{0} \otimes \cdots \otimes b_{j-1} \otimes 1 \otimes b_{j} \otimes \cdots \otimes b_{q}
$$

for $b_{0}, \ldots, b_{q} \in \mathbb{B}(i)^{*}$. Since $\mathbb{G}(i) \cong G_{n+1} \times S_{n}(i)$ as an $S_{n}(i)$-space, and $D^{q}$ is a finitely generated free $\mathbb{A}^{*}$-module, we see that $J^{q}(i, W) \cong I^{q}(W) \otimes D^{q}$. Then the differential $d^{q}: J^{q}(i, W) \rightarrow J^{q+1}(i, W)$ corresponds to

$$
d^{q}: I^{q}(i, W) \otimes \mathbb{B}(i)^{* \otimes(q+1)} \rightarrow I^{q+1}(i, W) \otimes \mathbb{B}(i)^{* \otimes(q+2)}
$$

given by

$d^{q}\left(f \otimes b_{0} \otimes \cdots \otimes b_{q}\right)\left(g_{0}, \ldots, q_{q+1}\right)$

$=\sum_{j=0}^{q+1}(-1)^{j} f\left(g_{0}, \ldots, g_{j-1}, g_{j+1}, \ldots, g_{q+1}\right) \otimes b_{0} \otimes \cdots \otimes b_{j-1} \otimes 1 \otimes b_{j} \otimes \cdots \otimes b_{q}$.

Proposition 5.12. For $p>0$ and $q \geq 0$, we have

$$
H_{c}^{p}\left(G_{n+1} ; J^{q}(i, W)\right)=0 \quad \text { and } \quad H_{c}^{0}\left(G_{n+1} ; J^{q}(i, W)\right)=C_{c}^{q}\left(G_{n+1} ; \mathbb{A}^{*}(W)\right) .
$$

The sequence $0 \rightarrow \mathbb{A}^{*}(W) \stackrel{d^{-1}}{\rightarrow} J^{0}(i, W) \stackrel{d^{0}}{\rightarrow} J^{1}(i, W) \stackrel{d^{2}}{\rightarrow} \cdots$ is a split exact sequence of topological $\mathbb{A}^{*}$-modules. Hence $J^{*}(i, W)$ is an acyclic resolution of $\mathbb{A}^{*}(W)$ with respect to $H_{c}^{*}\left(G_{n+1} ;-\right)$.

Proof. Let $M=\operatorname{Map}\left(S_{n}(i)^{q}, \mathbb{B}(i)^{*}(W)\right)$. We have an isomorphism $J^{q}(i, W) \cong$ $\operatorname{Map}_{c}\left(G_{n+1}^{q+1}, M\right)$ of topological $G_{n+1}$-modules. Since $M$ is a product of finite many copies of $\mathbb{A}^{*}(W)$, we can write $M=\lim _{\lambda} \mathbb{A}^{*} N_{\lambda}$ with finitely generated $N_{\lambda}$. Then the first assertion follows from Proposition 5.4. There is a continuous map $\varepsilon$ : $\mathbb{B}^{*}(i) \rightarrow \mathbb{A}^{*}$ of topological $\mathbb{A}^{*}$-modules such that $\varepsilon \circ \eta=1$, where $\eta: \mathbb{A}^{*} \rightarrow \mathbb{B}^{*}(i)$ is the unit. Define a map $s^{q}: I^{q}(i, W) \otimes \mathbb{B}(i)^{* \otimes(q+1)} \rightarrow I^{q-1}(i, W) \otimes \mathbb{B}(i)^{* \otimes q}$ by $s^{q}\left(f \otimes b_{0} \otimes \cdots \otimes b_{q}\right)\left(g_{0}, \ldots, g_{q-1}\right)=f\left(e, g_{0}, \ldots, g_{q-1}\right) \otimes \varepsilon\left(b_{0}\right) b_{1} \otimes \cdots \otimes b_{q}$.

Then we can verify that $\left\{s^{q}\right\}_{q \geq 0}$ gives a desired splitting.

We consider the double complexes $C_{c}^{*}\left(G_{n+1} ; I^{*}(W)\right)$ and $C_{c}^{*}\left(G_{n+1} ; J^{*}(i, W)\right)$. The canonical inclusion $\mathbb{A}^{*}(W) \rightarrow \mathbb{B}(i)^{*}(W)$ and the projection $\mathbb{G}(i) \rightarrow G_{n+1}$ induce a cochain map $I^{*}(W) \rightarrow J^{*}(i, W)$, which is equivariant under the actions of $G_{n+1}$. Hence we obtain a map of double complexes

$$
C_{c}^{*}\left(G_{n+1} ; I^{*}(W)\right) \longrightarrow C_{c}^{*}\left(G_{n+1} ; J^{*}(i, W)\right) .
$$


We denote by $\operatorname{Tot}^{*} C^{*, *}$ the total cochain complex of a double complex $C^{*, *}$.

Lemma 5.13. The cochain map

$$
\operatorname{Tot}^{*} C_{c}^{*}\left(G_{n+1} ; I^{*}(W)\right) \rightarrow \operatorname{Tot}^{*} C_{c}^{*}\left(G_{n+1} ; J^{*}(i, W)\right)
$$

is a quasi-isomorphism.

Proof. This follows from the fact that the map (5-3) induces an isomorphism on cohomology groups on the second index by Lemma 5.11 and Proposition 5.12.

Since the invariant subcomplex $I^{*}(W)^{G_{n+1}}$ is isomorphic to $C_{c}^{*}\left(G_{n+1} ; \mathbb{A}^{*}(W)\right)$, there is a cochain map

$$
C_{c}^{*}\left(G_{n+1} ; \mathbb{A}^{*}(W)\right) \longrightarrow \operatorname{Tot}^{*} C_{c}^{*}\left(G_{n+1} ; I^{*}(W)\right) .
$$

Since the invariant subcomplex $J^{*}(i, W)^{G_{n+1}}$ is isomorphic to $C_{c}^{*}\left(\mathbb{G}(i) ; \mathbb{B}(i)^{*}(W)\right)$, there is a cochain map

$$
C_{c}^{*}\left(\mathbb{G}(i) ; \mathbb{B}(i)^{*}(W)\right) \longrightarrow \operatorname{Tot}^{*} C_{c}^{*}\left(G_{n+1} ; J^{*}(i, W)\right) .
$$

Then we obtain the commutative diagram of cochain complexes

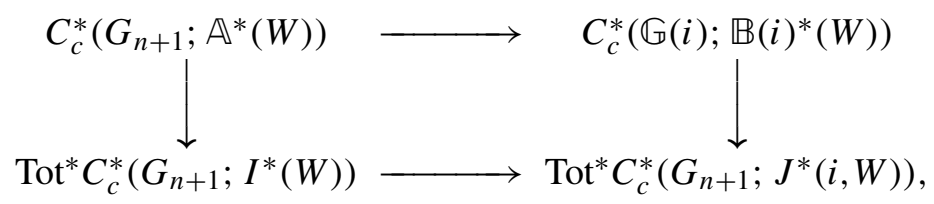

where the top horizontal arrow induces the inflation map

$$
H_{c}^{*}\left(G_{n+1} ; \mathbb{A}^{*}(W)\right) \longrightarrow H_{c}^{*}\left(\mathbb{G}(i) ; \mathbb{B}(i)^{*}(W)\right) .
$$

Lemma 5.14. The vertical arrows in the diagram (5-4) are quasi-isomorphisms.

Proof. By Lemma 5.11, the cohomology group of $C_{c}^{*}\left(G_{n+1} ; I^{*}(W)\right)$ on the first index is isomorphic to $C_{c}^{*}\left(G_{n+1} ; \mathbb{A}^{*}(W)\right)$. Hence the left vertical arrow is a quasiisomorphism. By Proposition 5.12, the cohomology group of $C_{c}^{*}\left(G_{n+1} ; J^{*}(i, W)\right)$ on the first index is isomorphic to $C_{c}^{*}\left(\mathbb{G}(i) ; \mathbb{B}(i)^{*}(W)\right)$. Hence the right vertical arrow is a quasi-isomorphism.

Corollary 5.15. The inflation map $H_{c}^{*}\left(G_{n+1} ; \mathbb{A}^{*}(W)\right) \longrightarrow H_{c}^{*}\left(\mathbb{G}(i) ; \mathbb{B}(i)^{*}(W)\right)$ is an isomorphism for any spectrum $W$ and any $i \geq 0$.

Proof of Theorem 5.8. Corollary 5.15 implies that the morphism (5-2) of spectral sequences is an isomorphism from the $E_{2}$-terms onward. Hence the inflation map (5-1) is an isomorphism. 
Remark 5.16. Let $\Lambda$ be an essentially small cofiltered category. For a system $\left\{N_{\lambda}\right\}_{\lambda \in \Lambda}$ of finitely generated twisted $E_{n+1}^{*}-G_{n+1}$-modules, we set $M=\lim _{\lambda_{\lambda}} \mathbb{A}^{*} N_{\lambda}$ and $\mathbb{B}^{*} M=\lim _{\lambda} \mathbb{B}^{*} \otimes_{\mathbb{A}^{*}} \mathbb{A}^{*} N_{\lambda}$. By the same method as above, we can define $\boldsymbol{H}_{c}^{*}\left(\mathbb{G} ; \mathbb{B}^{*} M\right)$ and show that there is an isomorphism

$$
H_{c}^{*}\left(G_{n+1} ; M\right) \stackrel{\cong}{\rightrightarrows} \boldsymbol{H}_{c}^{*}\left(\mathbb{G} ; \mathbb{B}^{*} M\right) .
$$

\section{Morphism of spectral sequences}

In this section we construct a natural morphism of spectral sequences from the $K(n)$-local $E_{n}$-Adams spectral sequence to the $K(n)$-localization of the $K(n+1)$ local $E_{n+1}$-Adams spectral sequence.

Let $B P$ be the Brown-Peterson spectrum at $p$. We denote by $B P^{\wedge s}$ the smash product of $s$ copies of $B P$ :

$$
B P^{\wedge s}=\overbrace{B P \wedge \cdots \wedge B P}^{s} .
$$

The commutative ring spectrum structure on $B P$ makes $B P^{\wedge \bullet+1}=\left\{B P^{\wedge s+1}\right\}_{s \geq 0}$ a cosimplicial object in the $p$-local stable homotopy category with augmentation $S_{(p)}^{0} \stackrel{\varepsilon}{\rightarrow} B P^{\wedge \bullet+1}$. Then the associated cochain complex

$$
* \rightarrow S_{(p)}^{0} \stackrel{\varepsilon}{\longrightarrow} B P \stackrel{d}{\longrightarrow} B P^{\wedge 2} \stackrel{d}{\longrightarrow} B P^{\wedge 3} \stackrel{d}{\longrightarrow} \cdots
$$

is a $p$-local $B P$-Adams resolution of $S_{(p)}^{0}$ in the sense of [Miller 1981; Devinatz and Hopkins 2004]. We denote by $\operatorname{Res}\left(B P ; S_{(p)}^{0}\right)$ the sequence (6-1). Then $\operatorname{Res}\left(B P ; S_{(p)}^{0}\right)$ gives us a diagram of exact triangles

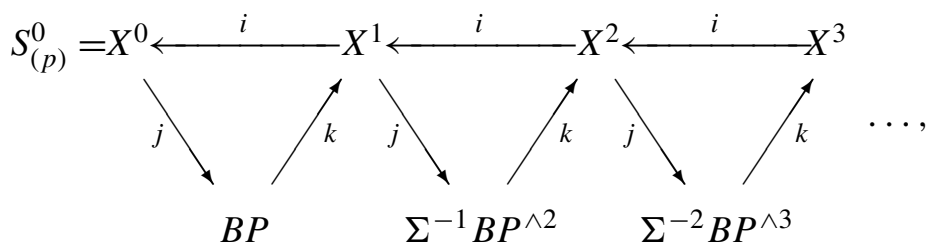

where $k$ has degree -1 and $j k=d$. We denote by $\operatorname{Ad}\left(B P ; S_{(p)}^{0}\right)$ the diagram of exact triangles (6-2).

By applying the $K(n)$-localization functor to the augmented cosimplicial commutative ring spectrum $S_{(p)}^{0} \stackrel{\varepsilon}{\rightarrow} B P^{\wedge \bullet+1}$, we obtain an augmented cosimplicial $K(n)$-local commutative ring spectrum $L_{K(n)} S^{0} \stackrel{\varepsilon}{\rightarrow} L_{K(n)} B P^{\wedge \bullet+1}$, and the associated augmented cochain complex

$$
* \rightarrow L_{K(n)} S^{0} \stackrel{\varepsilon}{\longrightarrow} L_{K(n)} B P \stackrel{d}{\longrightarrow} L_{K(n)} B P^{\wedge 2} \stackrel{d}{\longrightarrow} L_{K(n)} B P^{\wedge 3} \stackrel{d}{\longrightarrow} \cdots
$$


We denote by $L_{K(n)} \operatorname{Res}\left(B P ; S_{(p)}^{0}\right)$ the sequence (6-3).

Proposition 6.1. The sequence $L_{K(n)} \operatorname{Res}\left(B P ; S_{(p)}^{0}\right)$ is a $K(n)$-local $E_{n}$-Adams resolution of $L_{K(n)} S^{0}$.

Proof. To prove the proposition, it suffices to show that $L_{K(n)} B P^{\wedge s}$ is $E_{n}$-injective for $s>0$ and the sequence (6-3) is $E_{n}$-exact. By [Hovey and Sadofsky 1999, Theorem B], $L_{K(n)} B P$ is a coproduct of (suspensions of) $L_{K(n)} E(n)$ 's in the $K(n)$-local category. Since $L_{K(n)} E(n)$ is a direct summand of $E_{n}, L_{K(n)} B P$ is $E_{n}$-injective. Hence $L_{K(n)} B P^{\wedge s}$ is $E_{n}$-injective for $s>0$. To prove that the sequence (6-3) is $E_{n}$-exact, it is sufficient to show that the sequence (6-3) smashing with $E_{n}$ is a split exact sequence. There is a canonical ring spectrum map $\eta: L_{K(n)} B P \rightarrow E_{n}$. Then the following map

$L_{K(n)}\left(E_{n} \wedge B P^{\wedge s+1}\right) \stackrel{1 \wedge \eta \wedge 1^{\wedge s}}{\longrightarrow} L_{K(n)}\left(E_{n} \wedge E_{n} \wedge B P^{\wedge s}\right) \stackrel{m \wedge 1^{\wedge s}}{\longrightarrow} L_{K(n)}\left(E_{n} \wedge B P^{\wedge s}\right)$ for $s \geq 0$ gives a splitting, where $m$ is the multiplication of $E_{n}$.

The $K(n)$-localization functor gives a map of cosimplicial objects $B P^{\bullet+1} \rightarrow$ $E_{n}^{\bullet+1}$ covering the map $S_{(p)}^{0} \rightarrow L_{K(n)} S^{0}$. This induces a map

$$
L_{K(n)} \operatorname{Res}\left(B P ; S_{(p)}^{0}\right) \rightarrow \operatorname{Res}\left(E_{n} ; L_{K(n)} S^{0}\right)
$$

of cochain complexes and a map $L_{K(n)} \operatorname{Ad}\left(B P ; S^{0}\right) \rightarrow \operatorname{Ad}\left(E_{n} ; L_{K(n)} S^{0}\right)$ of diagrams of exact triangles. By Proposition 6.1, the map

$$
L_{K(n)} \operatorname{Res}\left(B P ; S_{(p)}^{0}\right) \rightarrow \operatorname{Res}\left(E_{n} ; L_{K(n)} S^{0}\right)
$$

is a cochain homotopy equivalence. Hence $L_{K(n)} \operatorname{Ad}\left(B P ; S^{0}\right) \rightarrow \operatorname{Ad}\left(E_{n} ; L_{K(n)} S^{0}\right)$ is an equivalence of diagram of exact triangles in an appropriate sense.

The canonical ring spectrum map $B P \rightarrow E_{n+1}$ induces a map of diagrams of exact triangles

$$
\operatorname{Ad}\left(B P ; S_{(p)}^{0}\right) \longrightarrow L_{K(n+1)} \operatorname{Ad}\left(B P ; S_{(p)}^{0}\right) \stackrel{\simeq}{\longrightarrow} \operatorname{Ad}\left(E_{n+1} ; L_{K(n+1)} S^{0}\right) .
$$

By applying the $K(n)$-localization functor to this map, we obtain a map of diagrams of exact triangles

$$
L_{K(n)} \operatorname{Ad}\left(B P ; S_{(p)}^{0}\right) \longrightarrow L_{K(n)} \operatorname{Ad}\left(E_{n+1} ; L_{K(n+1)} S^{0}\right) .
$$

Then this map of exact triangles implies the following theorem.

Theorem 6.2. For any spectrum $W$, there is a natural morphism of spectral sequences

$$
\varphi_{r}(W): L_{K(n)} E_{r}^{s, t}(W) \longrightarrow L_{K(n)} L_{K(n+1)} E_{r}^{s, t}(W),
$$

which converges to $\left[W, L_{K(n)} S^{0}\right]^{*} \rightarrow[W, \widehat{S}]^{*}$. 


\section{The inflation map}

In Section 6 we constructed a natural morphism

$$
\varphi_{r}(W): L_{K(n)} E_{r}^{*, *}(W) \rightarrow L_{K(n)} L_{K(n+1)} E_{r}^{*, *}(W)
$$

of spectral sequences for any spectrum $W$. In this section we construct a natural map $\theta(W): H_{c}^{*}\left(G_{n} ; E_{n}^{*}(W)\right) \rightarrow H_{c}^{*}\left(G_{n+1} ; \mathbb{A}^{*}(W)\right)$ by using the cohomology group $\boldsymbol{H}_{c}^{*}\left(\mathbb{G} ; \mathbb{B}^{*}(W)\right)$ in Section 5. Then we show that $\theta(W)$ coincides with $\varphi_{2}(W)$.

For a spectrum $W$, define cochain complexes $C_{B P}^{*, *}(W)$ and $L_{K(n)} C_{B P}^{*, *}(W)$ by

$$
\begin{aligned}
C_{B P}^{S, *}(W) & =\left[W, B P^{\wedge s+1}\right]^{*}, \\
L_{K(n)} C_{B P}^{s, *}(W) & =\left[W, L_{K(n)}\left(B P^{\wedge s+1}\right)\right]^{*} .
\end{aligned}
$$

The ring spectrum maps $B P \rightarrow L_{K(n)} B P \rightarrow E_{n}$ induce cochain maps

$$
C_{B P}^{*, *}(W) \rightarrow L_{K(n)} C_{B P}^{*, *}(W) \rightarrow C_{c}^{*}\left(G_{n} ; E_{n}^{*}(W)\right) .
$$

We shall describe the cochain map $C_{B P}^{*, *}(W) \rightarrow C_{c}^{*}\left(G_{n} ; E_{n}^{*}(W)\right)$ in terms of formal group laws. The universal deformation $F_{n}$ over $E_{n}^{0}$ induces a graded ring homomorphism $B P_{*} \rightarrow E_{n *}$. Recall that, for $g=(\gamma, s) \in \Gamma \ltimes S_{n}=G_{n}$, there is a unique isomorphism $t(g): F_{n} \rightarrow F_{n}^{g}$ over $E_{n}^{0}$, which is a lifting of the isomorphism $s: H_{n} \rightarrow H_{n}^{\gamma}=H_{n}$ over $\boldsymbol{F}$. For $g, h \in G_{n}$, we set $t(g, h)=t(h) \circ t(g)^{-1}: F_{n}^{g} \rightarrow F_{n}^{h}$. For a sequence $\boldsymbol{g}=\left(g_{0}, g_{1}, \ldots, g_{s}\right)$ of elements in $G_{n}$, we define a graded ring homomorphism

$$
t(\mathbf{g}): B P_{*}(B P)^{\otimes(s+1)} \longrightarrow E_{n *}
$$

to be the map representing the following string of isomorphisms of formal group laws

$$
F_{n} \stackrel{t\left(g_{0}\right)}{\longrightarrow} F_{n}^{g_{0}} \stackrel{t\left(g_{0}, g_{1}\right)}{\longrightarrow} F_{n}^{g_{1}} \stackrel{t\left(g_{1}, g_{2}\right)}{\longrightarrow} \cdots \stackrel{t\left(g_{s-1}, g_{s}\right)}{\longrightarrow} F_{n}^{g_{s}} .
$$

For a spectrum $W$, we denote by $\operatorname{ev}(g): C_{c}^{s}\left(G_{n} ; E_{n}^{*}(W)\right) \rightarrow E_{n}^{*}(W)$ the evaluation map at $\boldsymbol{g}=\left(g_{0}, g_{1}, \ldots, g_{s}\right)$. If $W$ is a finite spectrum, we denote its $S$-dual by $D W$. Then there are natural isomorphisms $B P^{-*}(W) \cong B P_{*}(D W)$ and $E_{n}^{-*}(W) \cong$ $E_{n *}(D W) \cong B P_{*}(D W) \otimes_{B P_{*}} E_{n *}$. In particular, we have

$$
C_{B P}^{s,-*}(W) \cong B P_{*}(D W) \otimes_{B P_{*}} B P_{*}(B P)^{\otimes s} .
$$

Lemma 7.1. Let $W$ be a finite spectrum. For a sequence $\boldsymbol{g}=\left(g_{0}, g_{1}, \ldots, g_{s}\right)$ of elements in $G_{n}$, the composition $C_{B P}^{s,-*}(W) \longrightarrow C_{c}^{s}\left(G_{n} ; E_{n}^{-*}(W)\right) \stackrel{\operatorname{ev}(g)}{\longrightarrow} E_{n}^{-*}(W)$ 
is given by

$$
\begin{gathered}
B P_{*}(D W) \otimes_{B P_{*}} B P_{*}(B P)^{\otimes s} \stackrel{\psi \otimes 1^{\otimes s}}{\longrightarrow} B P_{*}(D W) \otimes_{B P_{*}} B P_{*}(B P)^{\otimes(s+1)} \\
\stackrel{1 \otimes t(g)}{\longrightarrow} B P_{*}(D W) \otimes_{B P_{*}} E_{n *},
\end{gathered}
$$

where $\psi$ is the $B P_{*}(B P)$-comodule structure map of $B P_{*}(D W)$.

Proof. For $g \in G_{n}$, the ring spectrum map $g: E_{n} \rightarrow E_{n}$ induces a map $g^{-*}$ : $E_{n}^{-*}(W) \rightarrow E_{n}^{-*}(W)$. This map $g^{-*}$ is given by the composition

$$
\begin{gathered}
B P_{*}(D W) \otimes_{B P_{*}} E_{n *} \stackrel{\psi \otimes 1}{\longrightarrow} B P_{*}(D W) \otimes_{B P_{*}} B P_{*}(B P) \otimes_{B P_{*}} E_{n *} \\
\stackrel{1 \otimes t(g) \otimes g_{*}}{\longrightarrow} B P_{*}(D W) \otimes_{B P_{*}} E_{n *} .
\end{gathered}
$$

Next we consider the map $g_{0} \wedge \cdots \wedge g_{s}: E_{n}^{\wedge s+1} \rightarrow E_{n}^{\wedge s+1}$. This induces a map $\left(g_{0} \wedge \cdots \wedge g_{s}\right)^{-*}:\left(E_{n}^{\wedge s+1}\right)^{-*}(W) \rightarrow\left(E_{n}^{\wedge s+1}\right)^{-*}(W)$. Note that there is a natural isomorphism $\left(E_{n}^{\wedge s+1}\right)^{-*}(W) \cong B P_{*}(D W) \otimes_{B P_{*}} \pi_{*} E_{n}^{\wedge s+1}$ since $\pi_{*} E_{n}^{\wedge s+1}$ is Landweber exact. Then $\left(g_{0} \wedge \cdots \wedge g_{s}\right)^{-*}$ is given by

$$
\begin{aligned}
B P_{*}(D W) \otimes_{B P_{*}} \pi_{*} E_{n}^{\wedge s+1} \stackrel{\psi \otimes 1}{\longrightarrow} & B P_{*}(D W) \otimes_{B P_{*}} B P_{*}(B P) \otimes_{B P_{*}} \pi_{*} E_{n}^{\wedge s+1} \\
\stackrel{1 \otimes t\left(g_{0}\right) \otimes \pi_{*}\left(g_{0} \wedge \cdots \wedge g_{s}\right)}{\longrightarrow} & B P_{*}(D W) \otimes_{B P_{*}} E_{n *} \otimes_{E_{n *}} \pi_{*} E_{n}^{\wedge s+1} \\
\cong & B P_{*}(D W) \otimes_{B P_{*}} \pi_{*} E_{n}^{\wedge s+1} .
\end{aligned}
$$

The lemma follows from the fact that the composition

$$
C_{B P}^{s,-*}(W) \longrightarrow C_{c}^{s}\left(G_{n} ; E_{n}^{-*}(W)\right) \stackrel{\operatorname{ev}(g)}{\longrightarrow} E_{n}^{-*}(W)
$$

is induced by the map $B P^{\wedge s+1} \rightarrow E_{n}^{\wedge s+1} \stackrel{g_{0} \wedge \cdots \wedge g_{s}}{\longrightarrow} E_{n}^{\wedge s+1} \stackrel{m}{\longrightarrow} E_{n}$, where $m$ is the multiplication map of the ring spectrum $E_{n}$.

Next we construct a cochain map $C_{c}^{*}\left(G_{n} ; E_{n}^{*}(W)\right) \longrightarrow C_{c}^{*}\left(\mathbb{G}^{*} \mathbb{B}^{*}(W)\right)$, which induces a map $H_{c}^{*}\left(G_{n} ; E_{n}^{*}(W)\right) \longrightarrow \boldsymbol{H}_{c}^{*}\left(\mathbb{G} ; \mathbb{B}^{*}(W)\right)$.

Lemma 7.2. The ring spectrum map $I: E_{n} \rightarrow \mathbb{B}$ and the projection $\mathbb{G} \rightarrow G_{n}$ induce a cochain map $C_{c}^{*}\left(G_{n} ; E_{n}^{*}(W)\right) \longrightarrow C_{c}^{*}\left(\mathbb{G} ; \mathbb{B}^{*}(W)\right)$ for any spectrum $W$.

Proof. There are isomorphisms

$$
\begin{aligned}
C_{c}^{*}\left(G_{n} ; E_{n}^{*}(W)\right) & \cong \lim _{\lambda} \lim _{i} C_{c}^{*}\left(G(i), E_{n}^{*}\left(W_{\lambda}\right)\right), \\
C_{c}^{*}\left(\mathbb{G} ; \mathbb{B}^{*}(W)\right) & \cong \lim _{\lambda} \lim _{i} C_{c}^{*}\left(\mathbb{G}(i), \mathbb{B}(i)^{*}\left(W_{\lambda}\right)\right) .
\end{aligned}
$$

Then the canonical maps $E_{n}^{*}\left(W_{\lambda}\right) \rightarrow \mathbb{B}(i)^{*}\left(W_{\lambda}\right)$ and the projections $\mathbb{G}(i) \rightarrow G_{n}(i)$ induce the desired cochain map. 
Remark 7.3. Let $\Lambda$ be an essentially small cofiltered category. For a system $\left\{N_{\lambda}\right\}_{\lambda \in \Lambda}$ of finitely generated twisted $E_{n}^{*}-G_{n}$-modules annihilated by a power of the ideal $I_{n}$, we set $N=\lim _{\lambda} N_{\lambda}$ and $\mathbb{B}^{*} N=\varlimsup_{\lambda_{\lambda}} \mathbb{B}^{*} \otimes_{E_{n}^{*}} N_{\lambda}$. By the same method as above, we can obtain a cochain map $C_{c}^{*}\left(G_{n} ; N\right) \rightarrow \boldsymbol{C}_{c}^{*}\left(\mathbb{G} ; \mathbb{B}^{*} N\right)$.

Recall that in Section 5 we defined a cochain map $C_{c}^{*}\left(G_{n+1} ; \mathbb{A}^{*}(W)\right) \longrightarrow$ $\boldsymbol{C}_{c}^{*}\left(\mathbb{G} ; \mathbb{B}^{*}(W)\right)$, which induces an isomorphism of cohomology groups

$$
H_{c}^{*}\left(G_{n+1} ; \mathbb{A}^{*}(W)\right) \stackrel{\cong}{\rightarrow} \boldsymbol{H}_{c}^{*}\left(\mathbb{G} ; \mathbb{B}^{*}(W)\right)
$$

by Theorem 5.8. We define a map

$$
\theta(W): H_{c}^{*}\left(G_{n} ; E_{n}^{*}(W)\right) \longrightarrow H_{c}^{*}\left(G_{n+1} ; \mathbb{A}^{*}(W)\right)
$$

by the composition

$$
H_{c}^{*}\left(G_{n} ; E_{n}^{*}(W)\right) \longrightarrow \boldsymbol{H}_{c}^{*}\left(\mathbb{G} ; \mathbb{B}^{*}(W)\right) \stackrel{\cong}{\longleftarrow} H_{c}^{*}\left(G_{n+1} ; \mathbb{A}^{*}(W)\right),
$$

where the first map is induced by the cochain map in Lemma 7.2.

In the rest of this section we compare $\theta(W)$ to $\varphi_{2}(W)$. The ring spectrum maps $B P \rightarrow L_{K(n)} B P \rightarrow L_{K(n)} E_{n+1}=\mathbb{A}$ induce cochain maps

$$
C_{B P}^{*, *}(W) \rightarrow L_{K(n)} C_{B P}^{*, *}(W) \rightarrow C_{c}^{*}\left(G_{n+1} ; \mathbb{A}^{*}(W)\right) .
$$

We consider the following diagram of cochain complexes

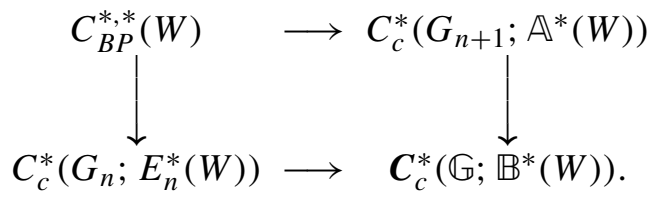

This diagram is not commutative but we shall show that it is cochain homotopy commutative for finite spectra $W$ by constructing a natural cochain homotopy.

Lemma 7.4. If $W$ is a finite spectrum, then the diagram (7-2) is cochain homotopy commutative.

Proof. Let $\pi: \mathbb{G} \rightarrow G_{n}$ be the projection. For $g, h \in \mathbb{G}$, we have an isomorphism of formal group laws $t(\pi(g), \pi(h)): F_{n}^{\pi(g)} \rightarrow F_{n}^{\pi(h)}$ over $E_{n}^{0}$. If we regard $t(\pi(g), \pi(h))$ as a power series over $\mathbb{B}^{0}$, then we obtain an isomorphism of formal group laws $t(g, h): F_{n}^{g} \rightarrow F_{n}^{h}$ over $\mathbb{B}^{0}$. In the same way we obtain an isomorphism of formal group laws $u(g, h): F_{n+1}^{g} \rightarrow F_{n+1}^{h}$ over $\mathbb{B}^{0}$. Recall that there is an isomorphism of formal group laws $\Phi: F_{n+1} \rightarrow F_{n}$ over $\mathbb{B}^{0}$. For a sequence $\boldsymbol{g}=\left(g_{0}, g_{1}, \ldots, g_{s}\right)$ of elements in $\mathbb{G}$, consider the following diagram of formal 
groups laws and isomorphisms over $\mathbb{B}^{0}$

$$
\begin{aligned}
F_{n+1} \stackrel{u\left(g_{0}\right)}{\longrightarrow} F_{n+1}^{g_{0}} \stackrel{u\left(g_{0}, g_{1}\right)}{\longrightarrow} F_{n+1}^{g_{1}} \longrightarrow \cdots \longrightarrow & F_{n+1}^{g_{i}} \\
& \downarrow^{\Phi^{g_{i}}} \\
& F_{n}^{g_{i}} \stackrel{t\left(g_{i}, g_{i+1}\right)}{\longrightarrow} F_{n}^{g_{i+1}} \longrightarrow \cdots \longrightarrow F_{n}^{g_{s}} .
\end{aligned}
$$

This diagram induces a graded ring homomorphism $T_{i}(\boldsymbol{g}): B P_{*}(B P)^{\otimes(s+2)} \rightarrow \mathbb{B}_{*}$. We fix an isomorphism between $\mathbb{B}^{-*}(W)$ and $B P_{*}(D W) \otimes_{B P_{*}} \mathbb{B}_{*}$, where $\mathbb{B}_{*}$ is a $B P_{*}$-module through the graded ring homomorphism $B P_{*} \rightarrow \mathbb{B}_{*}$ classifying the $p$-typical formal group law $F_{n+1}$. We define a map $C_{B P}^{s+1,-*}(W) \rightarrow \mathbb{B}^{-*}(W)$ by

$$
\begin{aligned}
& B P_{*}(D W) \otimes_{B P_{*}} B P_{*}(B P)^{\otimes(s+1)} \stackrel{\psi \otimes 1^{\otimes(s+1)}}{\longrightarrow} B P_{*}(D W) \otimes_{B P_{*}} B P_{*}(B P)^{\otimes(s+2)} \\
& \stackrel{1 \otimes T_{i}(\mathrm{~g})}{\longrightarrow} B P_{*}(D W) \otimes_{B P_{*}} \mathbb{B}_{*} .
\end{aligned}
$$

This map extends to a map

$$
S_{i}: C_{B P}^{s+1, *}(W) \longrightarrow \lim _{i} \operatorname{Map}_{c}\left(\mathbb{G}(i)^{s+1}, \mathbb{B}(i)^{*}(W)\right)^{\mathbb{G}(i)}=C_{c}^{s}\left(\mathbb{G} ; \mathbb{B}^{*}(W)\right) .
$$

We shall verify that $\sum_{i=0}^{s}(-1)^{i} S_{i}$ is a desired cochain homotopy. First note that the map $E_{n}^{-*}(W) \rightarrow \mathbb{B}^{-*}(W) \cong B P_{*}(D W) \otimes_{B P_{*}} \mathbb{B}_{*}$ is given by

$$
\begin{gathered}
B P_{*}(D W) \otimes_{B P_{*}} E_{n *} \stackrel{\psi \otimes 1}{\longrightarrow} B P_{*}(D W) \otimes_{B P_{*}} B P_{*}(B P) \otimes_{B P_{*}} E_{n *} \\
\stackrel{1 \otimes \Phi \otimes I_{*}}{\longrightarrow} B P_{*}(D W) \otimes_{B P_{*}} \mathbb{B}_{*},
\end{gathered}
$$

where $\Phi: B P_{*}(B P) \rightarrow \mathbb{B}_{*}$ is the graded ring homomorphism classifying the isomorphism $\Phi: F_{n+1} \rightarrow F_{n}$, and $I_{*}: E_{n *} \rightarrow \mathbb{B}_{*}$ is the induced map by the ring spectrum map $I$. Let $a^{*}$ be the cochain map $C_{B P}^{*, *}(W) \rightarrow C_{c}^{*}\left(G_{n} ; E_{n}^{*}(W)\right) \rightarrow C_{c}^{*}\left(\mathbb{G} ; \mathbb{B}^{*}(W)\right)$ and let $b^{*}$ be the cochain map $C_{B P}^{*, *}(W) \rightarrow C_{c}^{*}\left(G_{n+1} ; E_{n+1}^{*}(W)\right) \rightarrow C_{c}^{*}\left(\mathbb{G} ; \mathbb{B}^{*}(W)\right)$. We see that $\operatorname{ev}(\boldsymbol{g}) \circ a^{s}$ is given by

$$
\begin{aligned}
B P_{*}(D W) \otimes_{B P_{*}} B P_{*}(B P)^{\otimes s} & \stackrel{\psi \otimes 1^{\otimes s}}{\longrightarrow} B P_{*}(D W) \otimes_{B P_{*}} B P_{*}(B P)^{\otimes(s+1)} \\
& \stackrel{1 \otimes U(g)}{\longrightarrow} B P_{*}(D W) \otimes_{B P_{*}} \mathbb{B}_{*},
\end{aligned}
$$

where $U(\boldsymbol{g})$ is the graded ring homomorphism classifying the following string of isomorphisms of formal group laws

$$
F_{n+1} \stackrel{t\left(g_{0}\right) \circ \Phi}{\longrightarrow} F_{n}^{g_{0}} \stackrel{t\left(g_{0}, g_{1}\right)}{\longrightarrow} F_{n}^{g_{1}} \stackrel{t\left(g_{1}, g_{2}\right)}{\longrightarrow} \cdots \stackrel{t\left(g_{s-1}, g_{s}\right)}{\longrightarrow} F_{n}^{g_{s}} .
$$


In the cosimplicial module $C_{B P}^{\bullet, *}(W)$, the map $d_{i}: C_{B P}^{S,-*}(W) \rightarrow C_{B P}^{s+1,-*}(W)$ is given by

$$
d_{i}= \begin{cases}\psi \otimes 1^{\otimes s} & \text { if } i=0, \\ 1 \otimes 1^{\otimes(i-1)} \otimes \Delta \otimes 1^{\otimes(s-i)} & \text { if } 1 \leq i \leq s, \\ 1 \otimes 1^{\otimes s} \otimes \eta_{L} & \text { if } i=s+1,\end{cases}
$$

where $\triangle: B P_{*}(B P) \rightarrow B P_{*}(B P)^{\otimes 2}$ is the comultiplication, and $\eta_{L}: B P_{*} \rightarrow$ $B P_{*}(B P)$ is the left unit. Then we see that

$$
\begin{aligned}
S_{0} \circ d_{0} & =a^{s}, & & \\
S_{i} \circ d_{j} & =d_{j} \circ S_{i-1} & & \text { for } 0 \leq j<i \leq s, \\
S_{i-1} \circ d_{i} & =S_{i} \circ d_{i} & & \text { for } 0<i \leq s, \\
S_{i} \circ d_{j} & =d_{j-1} \circ S_{i} & & \text { for } 0 \leq i<j-1 \leq s, \\
S_{s} \circ d_{s+1} & =b^{s} . & &
\end{aligned}
$$

This implies that

$$
\sum_{i=0}^{s}(-1)^{i} S_{i} \circ \sum_{j=0}^{s+1}(-1)^{j} d_{j}+\sum_{j=0}^{s}(-1)^{j} d_{j} \circ \sum_{i=0}^{s-1}(-1)^{i} S_{i}=a^{s}-b^{s} .
$$

This completes the proof.

For a spectrum $W$, we have a similar diagram of cochain complexes

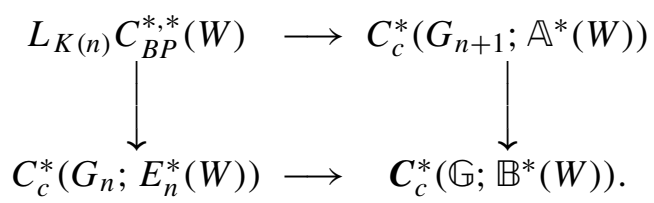

When $W$ is a finite spectrum, we let $S(W): C_{B P}^{*, *}(W) \rightarrow C_{c}^{*-1}\left(\mathbb{G} ; \mathbb{B}^{*}(W)\right)$ be the cochain homotopy constructed in the proof of Lemma 7.4. Then $S(W)$ extends to a cochain homotopy $L_{K(n)} S(W): L_{K(n)} C_{B P}^{*, *}(W) \rightarrow C_{c}^{*-1}\left(\mathbb{G} ; \mathbb{B}^{*}(W)\right)$, which makes the diagram (7-3) homotopy commutative.

Proposition 7.5. For any spectrum $W$, the diagram (7-3) is cochain homotopy commutative.

Proof. Since the cochain homotopy $L_{K(n)} S(W)$ is natural for finite spectra $W$, we obtain a cochain homotopy $\lim _{\lambda} L_{K(n)} S\left(W_{\lambda}\right):$

$$
\lim _{\lambda} L_{K(n)} C_{B P}^{*, *}\left(W_{\lambda}\right) \longrightarrow \lim _{\lambda} C_{c}^{*-1}\left(\mathbb{G} ; \mathbb{B}^{*}\left(W_{\lambda}\right)\right)=C_{c}^{*-1}\left(\mathbb{G} ; \mathbb{B}^{*}(W)\right),
$$


where the inverse limits are taken over $\lambda \in \Lambda(W)$. Then the composition with the cochain map $L_{K(n)} C_{B P}^{*, *}(W) \longrightarrow \lim _{\lambda} L_{K(n)} C_{B P}^{*, *}\left(W_{\lambda}\right)$ makes the diagram (7-3) cochain homotopy commutative.

Theorem 7.6. The map

$$
\theta(W): H_{c}^{*}\left(G_{n} ; E_{n}^{*}(W)\right) \rightarrow H_{c}^{*}\left(G_{n+1} ; E_{n+1}^{*}(W)\right)
$$

coincides with the map $\varphi_{2}(W)$ for any spectrum $W$.

Proof. In the diagram (7-3) the left vertical arrow is a quasi-isomorphism by Proposition 6.1. So is the right vertical arrow, by Theorem 5.8. The theorem follows because the top horizontal arrow induces the map $\varphi_{2}(W)$ and the bottom horizontal arrow induces the map $\theta(W)$.

\section{Nontriviality of the image of $\zeta_{n}$}

In this section we prove Theorem 8.1 as an application of the results in this note. By the Hopkins-Miller theorem [Devinatz and Hopkins 2004, Theorem 6], we know that there exists a nontrivial element $\zeta_{n} \in \pi_{-1}\left(L_{K(n)} S^{0}\right)$, which is represented by the reduced norm map of $G_{n}$ in the $E_{2}$-term of the $K(n)$-local $E_{n}$-Adams spectral sequence. The $K(n)$-localization of the $K(n+1)$-localization map $S^{0} \rightarrow L_{K(n+1)} S^{0}$ induces a map $L_{K(n)} S^{0} \rightarrow L_{K(n)} L_{K(n+1)} S^{0}$. In this section we show that the image of $\zeta_{n}$ under the map $\pi_{*}\left(L_{K(n)} S^{0}\right) \rightarrow \pi_{*}\left(L_{K(n)} L_{K(n+1)} S^{0}\right)$ is nontrivial as an application of Theorems 4.7 and 5.8.

By Theorem 6.2, we have a morphism of spectral sequences

$$
\varphi_{r}=\varphi_{r}\left(S^{0}\right): L_{K(n)} E_{r}^{*, *}\left(S^{0}\right) \longrightarrow L_{K(n)} L_{K(n+1)} E_{r}^{*, *}\left(S^{0}\right),
$$

which converges to $\pi_{*}\left(L_{K(n)} S^{0}\right) \rightarrow \pi_{*}\left(L_{K(n)} L_{K(n+1)} S^{0}\right)$. Then $\varphi_{2}$ is identified with the inflation map

$$
\theta=\theta\left(S^{0}\right): H_{c}^{*}\left(G_{n} ; E_{n}^{*}\right) \longrightarrow H_{c}^{*}\left(G_{n+1} ; \mathbb{A}^{*}\right)
$$

by Theorem 5.8. The reduced norm map of $G_{n}$ defines an element $z_{n} \in H_{c}^{1}\left(G_{n} ; E_{n}^{0}\right)$ which represents $\zeta_{n} \in \pi_{-1}\left(L_{K(n)} S^{0}\right)$. We set $w_{n}=\theta\left(z_{n}\right) \in H_{c}^{1}\left(G_{n+1} ; \mathbb{A}^{0}\right)$, and denote by $\omega_{n}$ the image of $\zeta_{n}$ under the map $\pi_{*}\left(L_{K(n)} S^{0}\right) \rightarrow \pi_{*}\left(L_{K(n)} L_{K(n+1)} S^{0}\right)$. Then $w_{n}$ is a permanent cycle and it represents $\omega_{n}$.

Theorem 8.1. $\omega_{n} \in \pi_{-1}\left(L_{K(n)} L_{K(n+1)} S^{0}\right)$ is nontrivial.

Proof. In [Torii 2003] we constructed a map

$$
\theta^{\prime}: H_{c}^{*}\left(G_{n} ; \boldsymbol{F}\left[w^{ \pm 1}\right]\right) \longrightarrow H_{c}^{*}\left(G_{n+1} ; \boldsymbol{F}\left(\left(u_{n}\right)\right)\left[u^{ \pm 1}\right]\right)
$$


Then there exists a commutative diagram

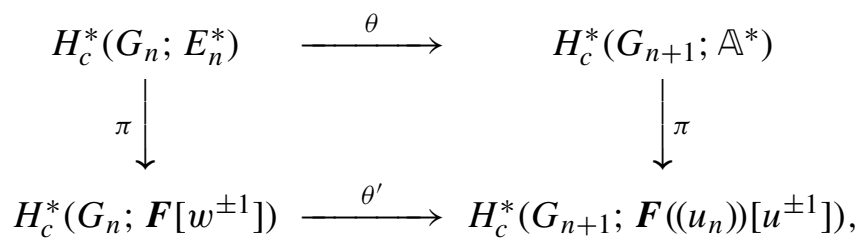

where the vertical arrows $\pi$ are canonical reduction maps. In [Torii 2005] we calculated the image of $\theta^{\prime}: H_{c}^{1}\left(G_{n} ; \boldsymbol{F}\left[w^{ \pm 1}\right]\right) \rightarrow H_{c}^{1}\left(G_{n+1} ; \boldsymbol{F}\left(\left(u_{n}\right)\right)\left[u^{ \pm 1}\right]\right)$, and we showed that $\theta^{\prime}\left(\pi\left(z_{n}\right)\right)$ is nontrivial. This implies that $\theta\left(z_{n}\right) \in H_{c}^{1}\left(G_{n+1} ; \mathbb{A}^{0}\right)$ is nontrivial. Since $\theta\left(z_{n}\right)$ is a permanent cycle and lies in the 1-line of the spectral sequence, it represents a nontrivial element in $\pi_{-1}\left(L_{K(n)} L_{K(n+1)} S^{0}\right)$.

\section{References}

[Boardman 1999] J. M. Boardman, "Conditionally convergent spectral sequences", pp. 49-84 in Homotopy invariant algebraic structures (Baltimore, MD, 1998), edited by J.-P. Meyer et al., Contemporary Mathematics 239, AMS, Providence, RI, 1999. MR 2000m:55024 Zbl 0947.55020

[Bousfield and Kan 1972] A. K. Bousfield and D. M. Kan, Homotopy limits, completions and localizations, Lecture Notes in Mathematics 304, Springer, Berlin, 1972. MR 51 \#1825 Zbl 0259.55004

[Chase et al. 1965] S. U. Chase, D. K. Harrison, and A. Rosenberg, "Galois theory and Galois cohomology of commutative rings”, Mem. Am. Math. Soc. 52 (1965), 15-33. MR 33 \#4118 Zbl 0143.05902

[Devinatz and Hopkins 2004] E. S. Devinatz and M. J. Hopkins, "Homotopy fixed point spectra for closed subgroups of the Morava stabilizer groups", Topol. 43:1 (2004), 1-47. MR 2004i:55012 Zbl 1047.55004

[Greither 1992] C. Greither, Cyclic Galois extensions of commutative rings, Lecture Notes in Mathematics 1534, Springer, Berlin, 1992. MR 94k:11121 Zbl 0788.13003

[Hovey 1995] M. A. Hovey, "Bousfield localization functors and Hopkins' chromatic splitting conjecture”, pp. 225-250 in The Čech centennial (Boston, 1993), edited by M. Cenkl and H. R. Miller, Contemporary Mathematics 181, AMS, Providence, RI, 1995. MR 96m:55010 Zbl 0830.55004

[Hovey 1997] M. A. Hovey, " $v_{n}$-Elements in ring spectra and applications to bordism theory", Duke Math. J. 88:2 (1997), 327-356. MR 98d:55017 Zbl 0880.55006

[Hovey and Sadofsky 1999] M. A. Hovey and H. Sadofsky, "Invertible spectra in the $E(n)$-local stable homotopy category", J. Lond. Math. Soc. (2) 60:1 (1999), 284-302. MR 2000h:55017 Zbl 0947.55013

[Hovey and Strickland 1999] M. A. Hovey and N. P. Strickland, Morava K-theories and localisation, Mem. Am. Math. Soc. 139:666, 1999. MR 99b:55017 Zbl 0929.55010

[Hovey et al. 1997] M. A. Hovey, J. H. Palmieri, and N. P. Strickland, Axiomatic stable homotopy theory, Mem. Am. Math. Soc. 128:610, 1997. MR 98a:55017 Zbl 0881.55001

[Lam 1999] T. Y. Lam, Lectures on modules and rings, Graduate Texts in Mathematics 189, Springer, New York, 1999. MR 99i:16001 Zbl 0911.16001

[Miller 1981] H. R. Miller, "On relations between Adams spectral sequences, with an application to the stable homotopy of a Moore space", J. Pure Appl. Algebra 20:3 (1981), 287-312. MR 82f:55029 Zbl 0459.55012 
[Milne 1980] J. S. Milne, Étale cohomology, Princeton Mathematical Series 33, Princeton University Press, Princeton, NJ, 1980. MR 81j:14002 Zbl 0433.14012

[Morava 1985] J. Morava, "Noetherian localisations of categories of cobordism comodules", Ann. Math. (2) 121:1 (1985), 1-39. MR 86g:55004 Zbl 0572.55005

[Ravenel 1992] D. C. Ravenel, Nilpotence and periodicity in stable homotopy theory, Ann. Math. Studies 128, Princeton University Press, Princeton, NJ, 1992. MR 94b:55015 Zbl 0774.55001

[Serre 1994] J.-P. Serre, Cohomologie galoisienne, 5th ed., Lecture Notes in Math. 5, Springer, Berlin, 1994. Translated in Galois cohomology, Springer, Berlin, 2002. MR 96b:12010 Zbl 0812. 12002

[Torii 2003] T. Torii, "On degeneration of one-dimensional formal group laws and applications to stable homotopy theory”, Am. J. Math. 125:5 (2003), 1037-1077. MR 2004i:55006 Zbl 1046.55003

[Torii 2005] T. Torii, "On relations between 1-lines of Adams-Novikov spectral sequences modulo invariant prime ideals", Topol. Appl. 150:1-3 (2005), 33-57. MR 2005k:55026 Zbl 1069.55002

[Torii 2010a] T. Torii, “Comparison of Morava E-theories”, Math. Zeitschrift 266:4 (2010), 933951. MR 2729298 Zbl 1203.55004

[Torii 2010b] T. Torii, “On $E_{\infty}$-structure of the generalized Chern character", Bull. Lond. Math. Soc. 42:4 (2010), 680-690. MR 2669689 Zbl 05770958

Received April 3, 2009. Revised December 17, 2009.

TAKESHI TORII

DEPARTMENT OF MATHEMATICS

OKAYAMA UNIVERSITY

OKAYAMA 700-8530

JAPAN

torii@ @ath.okayama-u.ac.jp 


\title{
PACIFIC JOURNAL OF MATHEMATICS
}

\author{
http://www.pjmath.org \\ Founded in 1951 by
}

E. F. Beckenbach (1906-1982) and F. Wolf (1904-1989)

\section{EDITORS}

V. S. Varadarajan (Managing Editor)

Department of Mathematics

University of California

Los Angeles, CA 90095-1555

pacific@math.ucla.edu

Vyjayanthi Chari

Department of Mathematics

University of California

Riverside, CA 92521-0135

chari@math.ucr.edu

\section{Robert Finn}

Department of Mathematics Stanford University

Stanford, CA 94305-2125

finn@math.stanford.edu

Kefeng Liu

Department of Mathematics

University of California

Los Angeles, CA 90095-1555

liu@math.ucla.edu
Darren Long

Department of Mathematics

University of California

Santa Barbara, CA 93106-3080

long@math.ucsb.edu

Jiang-Hua Lu

Department of Mathematics

The University of Hong Kong

Pokfulam Rd., Hong Kong jhlu@maths.hku.hk

Alexander Merkurjev

Department of Mathematics University of California

Los Angeles, CA 90095-1555 merkurev@math.ucla.edu
Sorin Popa

Department of Mathematics

University of California

Los Angeles, CA 90095-1555

popa@math.ucla.edu

Jie Qing

Department of Mathematics

University of California

Santa Cruz, CA 95064

qing@ cats.ucsc.edu

Jonathan Rogawski

Department of Mathematics

University of California

Los Angeles, CA 90095-1555

jonr@math.ucla.edu

\section{PRODUCTION}

pacific@math.berkeley.edu

Silvio Levy, Scientific Editor Matthew Cargo, Senior Production Editor

ACADEMIA SINICA, TAIPEI

CALIFORNIA INST. OF TECHNOLOGY

INST. DE MATEMÁTICA PURA E APLICADA

KEIO UNIVERSITY

MATH. SCIENCES RESEARCH INSTITUTE

NEW MEXICO STATE UNIV.

OREGON STATE UNIV.

\section{SUPPORTING INSTITUTIONS}

STANFORD UNIVERSITY
UNIV. OF BRITISH COLUMBIA
UNIV. OF CALIFORNIA, BERKELEY
UNIV. OF CALIFORNIA, DAVIS
UNIV. OF CALIFORNIA, LOS ANGELES
UNIV. OF CALIFORNIA, RIVERSIDE
UNIV. OF CALIFORNIA, SAN DIEGO
UNIV. OF CALIF., SANTA BARBARA

UNIV. OF CALIF., SANTA CRUZ

UNIV. OF MONTANA

UNIV. OF OREGON

UNIV. OF SOUTHERN CALIFORNIA

UNIV. OF UTAH

UNIV. OF WASHINGTON

WASHINGTON STATE UNIVERSITY

These supporting institutions contribute to the cost of publication of this Journal, but they are not owners or publishers and have no responsibility for its contents or policies.

See inside back cover or www.pjmath.org for submission instructions.

The subscription price for 2011 is US \$420/year for the electronic version, and \$485/year for print and electronic.

Subscriptions, requests for back issues from the last three years and changes of subscribers address should be sent to Pacific Journal of Mathematics, P.O. Box 4163, Berkeley, CA 94704-0163, U.S.A. Prior back issues are obtainable from Periodicals Service Company, 11 Main Street, Germantown, NY 12526-5635. The Pacific Journal of Mathematics is indexed by Mathematical Reviews, Zentralblatt MATH, PASCAL CNRS Index, Referativnyi Zhurnal, Current Mathematical Publications and the Science Citation Index.

The Pacific Journal of Mathematics (ISSN 0030-8730) at the University of California, c/o Department of Mathematics, 969 Evans Hall, Berkeley, CA 94720-3840, is published monthly except July and August. Periodical rate postage paid at Berkeley, CA 94704, and additional mailing offices. POSTMASTER: send address changes to Pacific Journal of Mathematics, P.O. Box 4163, Berkeley, CA 94704-0163.

PJM peer review and production are managed by EditFLOW ${ }^{\mathrm{TM}}$ from Mathematical Sciences Publishers.

PUBLISHED BY PACIFIC JOURNAL OF MATHEMATICS

at the University of California, Berkeley 94720-3840

A NON-PROFIT CORPORATION

Typeset in IATEX

Copyright $(2011$ by Pacific Journal of Mathematics 


\section{PACIFIC JOURNAL OF MATHEMATICS}

Volume $250 \quad$ No. $2 \quad$ April 2011

Realizing profinite reduced special groups

VinCENT Astier and Hugo Mariano

On fibered commensurability

DANNy CALEGARI, Hongbin SUN and Shicheng WANG

On an overdetermined elliptic problem

LAURENT HaUswirTh, FrÉdÉRIC HÉLEIN and FranK PACARD

Minimal sets of a recurrent discrete flow

HATTAB HAWETE

Trace-positive polynomials

IGOR KLEP

Remarks on the product of harmonic forms

LiviU ORnea and Mihaela Pilca

Steinberg representation of GSp(4): Bessel models and integral representation of $L$-functions

Ameya Pitale

An integral expression of the first nontrivial one-cocycle of the space of long knots in $\mathbb{R}^{3}$

KEIICHI SAKAI

Burghelea-Haller analytic torsion for twisted de Rham complexes

GUANGXIANG SU

$K(n)$-localization of the $K(n+1)$-local $E_{n+1}$-Adams spectral sequences

TAKESHI TORII

Thompson's group is distorted in the Thompson-Stein groups

Claire Wladis

Parabolic meromorphic functions 